\title{
Dynamical Approximation Entropies and Topological Entropy in Operator Algebras
}

\author{
Dan Voiculescu ${ }^{\star}$ \\ Department of Mathematics, University of California Berkeley, California 94720, USA
}

Received: 25 April 1994 / in revised form 24 August 1994

\begin{abstract}
Dynamical entropy invariants, based on a general approximation approach are introduced for $\mathrm{C}^{*}$ - and $\mathrm{W}^{*}$-algebra automorphisms. This includes a noncommutative extension of topological entropy.
\end{abstract}

The Connes-Störmer entropy [4] and its generalization by Connes-NarnhoferThirring [3] (see [9] for a recent alternative approach) extend the entropy invariant of Kolmogorov to the context of $W^{*}$-algebra automorphisms. These entropies may be viewed as "observable"-entropies, i.e. they are based on the physics point of view of observing the quantum dynamical system via abelian models. Here we explore another route to entropy based on approximation (we also briefly discussed the approximation idea in [14]). One may think of approximation entropies as "growth"entropies, reflecting the mathematical idea of the growth of the algebra produced by the automorphism. The invariants we obtain are $\geqq$ the usual ones and we show equality for non-commutative Bernoulli shifts and in the commutative cases. Note also that "observable" entropy is essential in getting lower bounds for approximation entropies, while approximation entropies give useful upper bounds for "observable" entropy. Let us also mention from the beginning that the natural framework for Connes-Störmer. Connes-Narnhofer-Thirring and approximation entropies is that of algebras satisfying hyperfiniteness or nuclearity assumptions.

There are several reasons for studying approximation entropies. One motivation is the search for a non-commutative analogue to McMillan's theorem for the Connes-Störmer entropy. Proving equality of the Connes-Störmer entropy and of the approximation entropy for a given automorphism can be viewed as a kind of weak McMillan-type of theorem.

For approximation entropies, the entropy of a tensor product of two automorphisms is bounded by the sum of the entropies of the automorphisms. Powers' shifts, which are very far from asymptotically abelian have been shown recently by Narnhofer-Störmer-Thirring [15] to provide a counterexample to this tensor product property for the Connes-Störmer entropy. For these Powers' shifts the Connes-

^ Supported in part by Grant No. DMS 9204174 from the National Science Foundation. 
Störmer entropy and the approximation entropies are different. More generally, the lack of asymptotic abelianness appears to get in the way of observing the system via abelian models (a point of view held by $\mathrm{H}$. Narnhofer for some time) and therefore one should also expect McMillan-type results for the Connes-Störmer entropy only when asymptotic abelianness is satisfied.

Another reason for the approximation approach is that it provides a suitable definition of topological entropy in the non-commutative context. The definitions previously proposed $([5,13])$, based on the covering idea, have the drawback of not being invariants of the $C^{*}$-algebra automorphism, i.e. they require additional structure in order to function. To define topological entropy in the approximation approach, it suffices to replace the 2-norm defined by the invariant state by the uniform norm and one obtains a definition with adequate features.

We also think approximation is consistent with the spirit of [10] and [6].

We consider two kinds of approximation by finite-dimensional subalgebras and via completely positive maps. Multiplying these two possibilities by the two cases, that of an invariant state and the $C^{*}$-case (i.e. "measurable" amd "topological"), we get four approximation entropies. For McMillan-type results the stronger statements correspond to approximation by subalgebras. Our topological entropy is based on completely positive approximation in the $C^{*}$-case.

For automorphisms of non-commutative tori we show that their topological entropy is of the same order as the entropy of the corresponding classical system.

Another example where we compute the topological entropy is the inner automorphism for the implementing unitary of a topological Bernoulli shift in the crossed product. This also solves in the affirmative in the case of Bernoulli shifts with equal weights a question of E. Störmer in [11]. In an Appendix using the completely positive approximation entropy we solve the problem in [11] for general ergodic transformations.

Note that the approximation idea used here can be applied as well for automorphisms of other topological algebraic structures. As an example we briefly look at the Hilbert space case. The result is that the approximation entropy of a unitary operator coincides with the Connes-Störmer entropy of the Bogoliubov automorphism it defines.

Also more generally we define the entropy of a unitary representation for a certain class of amenable discrete groups. In the case of an i.c.c. group for a representation quasiequivalent with the regular representation we prove the entropy equals the von Neumann dimension.

The paper has nine sections (except the introduction). The first four sections deal with the four approximation entropies for operator algebras: first the two corresponding to subalgebra approximation $\left(W^{*}\right.$ - and $C^{*}$-case) and then the two cases ( $W^{*}$ and $C^{*}$ ) of completely positive approximation. Section 5 deals with the topological entropy of automorphisms of non-commutative tori. Section 6 is about the topological entropy of the inner automorphism implementing the topological Bernoulli shift in the crossed product. Section 7 and 8 deal with Hilbert space entropy, first the case of a unitary operator, then the case of a group representation. Section 9 computes the entropy of the inner automorphism implementing the topological Bernoulli shift in the crossed product. The last section entitled "Further Remarks" discusses variants of definitions and some of the open problems. There is also an Appendix about the solution to Störmer's problem in general. 


\section{Subalgebra Approximation the $W^{*}$-Case}

Let $M$ be a separable hyperfinite, finite von Neumann algebra with a faithful normal trace state $\tau$ and let $\left|x_{2}\right|=\left(\tau\left(x^{*} x\right)\right)^{1 / 2}$ be the associated 2-norm. By $\mathscr{P} f(M)$ we denote the finite subsets of $M$. If $\omega \in \mathscr{P} f(M)$ and $\mathscr{X} \subset M$ we shall write $\omega \subset_{\delta} \mathscr{X}$ if for every $a \in \omega$ there is $x \in \mathscr{X}$ such that $|a-x|_{2}<\delta$. Let further $\mathscr{F}(M)$ denote the unital finite-dimensional $C^{*}$-subalgebras of $M$. If $A \in \mathscr{F}(M)$ we denote by $\operatorname{dim}$ $A$ its dimension and by $\operatorname{rank} A$ its rank, i.e. the dimension of a maximal abelian self-adjoint subalgebra of $A$.

1.1. Definition. If $\omega \in \mathscr{P} f(M)$ and $\delta>0$ we define

$$
r_{\tau}(\omega ; \delta)=\inf \left\{\operatorname{rank} A \mid A \in \mathscr{F}(M), \omega \subset_{\delta} A\right\}
$$

the $\delta$-rank of $\omega$.

1.2. Definition. If $\alpha$ is an automorphism of $M$ such that $\tau \circ \alpha=\tau, \delta>0$ and $\omega \in \mathscr{P} f(M)$ we define

$$
\begin{aligned}
h a_{\tau}(\alpha, \omega ; \delta) & =\limsup _{n \rightarrow \infty} n^{-1} \log r_{\tau}\left(\omega \cup \alpha(\omega) \cup \ldots \cup \alpha^{n-1}(\omega) ; \delta\right), \\
h a_{\tau}(\alpha, \omega) & =\sup _{\delta>0} h a_{\tau}(\alpha, \omega ; \delta), \\
h a_{\tau}(\alpha) & =\sup \left\{h a_{\tau}(\alpha, \omega) \mid \omega \in \mathscr{P} f(M)\right\} .
\end{aligned}
$$

$h a_{\tau}(\alpha)$ will be called the approximation entropy of $\alpha$.

1.3. Proposition. If $k \in \mathbb{Z}$ then

$$
h a_{\tau}\left(\alpha^{k}\right)=|k| h a_{\tau}(\alpha)
$$

Proof. We first show $h a(\alpha)=h a\left(\alpha^{-1}\right)$. We have

$$
\begin{aligned}
r_{\tau}\left(\bigcup_{0 \leqq j \leqq n-1} \alpha^{j}(\omega) ; \delta\right) & =r_{\tau}\left(\alpha^{-n+1}\left(\bigcup_{0 \leqq j \leqq n-1} \alpha^{j}(\omega)\right) ; \delta\right) \\
& =r_{\tau}\left(\bigcup_{0 \leqq j \leqq n-1} \alpha^{-j}(\omega) ; \delta\right),
\end{aligned}
$$

and hence

$$
\begin{aligned}
h a_{\tau}(\alpha, \omega ; \delta) & =h a_{\tau}\left(\alpha^{-1}, \omega ; \delta\right), \\
h a_{\tau}(\alpha, \omega) & =h a_{\tau}\left(\alpha^{-1}, \omega\right), \\
h a_{\tau}(\alpha) & =h a_{\tau}\left(a^{-1}\right) .
\end{aligned}
$$

Thus we may assume $k>0$. We have

$$
\begin{aligned}
h a_{\tau}\left(\alpha^{k}, \omega ; \delta\right) & =\limsup _{n \rightarrow \infty} n^{-1} \log r_{\tau}\left(\bigcup_{0 \leqq j \leqq n-1} \alpha^{k j}(\omega) ; \delta\right) \\
& \leqq \sup _{n \rightarrow \infty} n^{-1} \log r_{\tau}\left(\bigcup_{0 \leqq j \leqq k(n-1)} \alpha^{j}(\omega) ; \delta\right) \\
& \leqq k h a_{\tau}(\alpha, \omega ; \delta),
\end{aligned}
$$


which implies $h a_{\tau}\left(\alpha^{k}\right) \leqq k h a_{\tau}(\omega)$.

For the reverse inequality, remark that if $\omega \in \mathscr{P} f(M)$ and

$$
\omega_{1}=\bigcup_{0 \leqq j \leqq k-1} \alpha^{j}(\omega)
$$

then

$$
r_{\tau}\left(\bigcup_{0 \leqq j \leqq n-1} \alpha^{j}(\omega) ; \delta\right) \leqq r_{\tau}\left(\bigcup_{0 \leqq j \leqq\left[\frac{n}{h}\right]} \alpha^{k j}\left(\omega_{1}\right) ; \delta\right)
$$

Hence

$$
\begin{aligned}
k h a_{\tau}(\alpha, \omega ; \delta) & \leqq h a_{\tau}\left(\alpha^{k}, \omega_{1} ; \delta\right), \\
k h a_{\tau}(\alpha, \omega) & \leqq h a_{\tau}\left(\alpha^{k}, \omega_{1}\right), \\
k h a_{\tau}(\alpha) & \leqq h a_{\tau}\left(\alpha^{k}\right)
\end{aligned}
$$

1.4. Proposition. Let $\omega_{j} \in \mathscr{P} f(M), j \in \mathbb{N}, \omega_{1} \subset \omega_{2} \subset \ldots$ be such that $\cup_{j \in \mathbb{N}}$ $\cup_{n \in \mathbb{Z}} \alpha^{n}\left(\omega_{j}\right)$ generates $M$ as a von Neumann algebra. Then

$$
h a_{\tau}(\alpha)=\sup _{j \in \mathbb{N}} h a_{\tau}\left(\alpha, \omega_{j}\right) .
$$

Proof. It suffices to show that given $\omega \in \mathscr{P} f(M)$ and $\delta>0$ there is $\delta_{1}>0$ and $\omega_{j}$ such that

$$
h a_{\tau}(\alpha, \omega ; \delta) \leqq h a_{\tau}\left(\alpha, \omega_{j} ; \delta_{1}\right) .
$$

In view of the assumptions there is $N \in \mathbb{N}$ so that

$$
\omega \subset_{\delta / 2} N \operatorname{co}\left(\mathbb{T}\left(\{1\} \cup \bigcup_{|k| \leqq N} \alpha^{k}\left(\omega_{j} \cup \omega_{j}^{*}\right)\right)^{N}\right),
$$

where $\omega_{j}^{*}=\left\{a^{*} \mid a \in \omega_{j}\right\}, \mathbb{T}=\{z \in \mathbb{C}|| z \mid=1\}$ and co denotes the convex hull. If $C=\max \left\{\|a\| \mid a \in \omega_{j}\right\}$ we easily see that if for some unital $B \in \mathscr{F}(M)$ we have

$$
\bigcup_{|k| \leqq N} \alpha^{k}\left(\omega_{j}\right) \subset_{\delta_{1}} B
$$

then

$$
N_{c o}\left(\mathbb{T}\left(\{1\} \cup \bigcup_{|k| \leqq N} \alpha^{k}\left(\omega_{j} \cup \omega_{j}^{*}\right)\right)^{N}\right) \subset_{\delta_{2}} B,
$$

where $\delta_{2}=N^{2}(2 C)^{N-1} \delta_{1}$. Thus choosing $\delta_{1}=2^{-1} N^{-2}(2 C)^{1-N} \delta_{2}$ we see that

$$
\bigcup_{|k| \leqq N} \alpha^{k}\left(\omega_{J}\right) \subset_{\delta_{1}} B
$$

implies $\omega \subset_{\delta} B$. In particular

$$
r_{\tau}\left(\bigcup_{0 \leqq k \leqq n-1} \alpha^{k}(\omega) ; \delta\right) \leqq r_{\tau}\left(\bigcup_{-N \leqq k \leqq N+n-1} \alpha^{k}\left(\omega_{j}\right) ; \delta_{1}\right),
$$

so that 


$$
h a_{\tau}(\alpha, \omega ; \delta) \leqq h a_{\tau}\left(\alpha, \alpha^{-N}\left(\omega_{j}\right) ; \delta_{1}\right)=h a_{\tau}\left(\alpha, \omega_{j} ; \delta_{1}\right)
$$

The notations $H\left(A_{1}, \ldots, A_{n}\right), H(A \mid B), H(A, \alpha), H(\alpha)$ are those of [4] for the entropy quantities defined by A. Connes and E. Störmer.

1.5. Proposition. If $A \in \mathscr{F}(M)$ and $\omega \in \mathscr{P} f(M)$ generates $A$ as a $C^{*}$-algebra, then

$$
H(A, \alpha) \leqq h a_{\tau}(\alpha, \omega)
$$

and

$$
H(\alpha) \leqq h a_{\tau}(\alpha)
$$

Proof. It is clearly sufficient to prove the first assertion.

For the given $A \in \mathscr{F}(M)$ and a given $\varepsilon>0$ by [4] there is $\delta>0$ such that if $B \in \mathscr{F}(M)$ and if for every $a \in A,\|a\| \leqq 1$ there is $b \in B$ such that $|a-b|_{2}<\delta$, then $H(A \mid B)<\varepsilon$. It is easily seen that in view of our assumptions there is $\delta_{1}>0$ such that

$$
\omega \subset_{\delta_{1}} B \Rightarrow H(A \mid B)<\varepsilon
$$

This also implies

$$
\alpha^{n}(\omega) \subset_{\delta_{1}} B \Rightarrow H\left(\alpha^{n}(A) \mid B\right)<\varepsilon
$$

Thus, if

$$
r_{\tau}\left(\omega \cup \cdots \cup \alpha^{n-1}(\omega) ; \delta_{1}\right)=r(n),
$$

there is $B_{n} \in \mathscr{F}(M)$, rank $B_{n}=r(n)$ so that $\alpha^{j}(\omega) \subset_{\delta_{1}} B_{n}$ for $0 \leqq j \leqq n-1$. This implies

$$
\begin{aligned}
H\left(A, \alpha(A), \ldots, \alpha^{n-1}(A)\right) & \leqq H\left(B_{n}\right)+H\left(A \mid B_{n}\right)+\cdots+H\left(a^{n-1}(A) \mid B_{n}\right) \\
& \leqq \log r(n)+n \varepsilon,
\end{aligned}
$$

so that

$$
H(A, \alpha) \leqq h a_{\tau}\left(\alpha, \omega ; \delta_{1}\right)+\varepsilon,
$$

and hence the desired conclusion.

1.6 Remark. It is obvious that the preceding proposition can be refined by replacing the lim sup in the definition of $h a_{\tau}(\alpha, \omega ; \delta)$ by a lim inf. This defines lower approximation entropy quantities:

$$
\begin{aligned}
\operatorname{lh} a_{\tau}(\alpha, \omega ; \delta) & =\liminf _{n \rightarrow \infty} n^{-1} \log r_{\tau}\left(\omega \cup \cdots \cup \alpha^{-1}(\omega) ; \delta\right), \\
\operatorname{lh} a_{\tau}(\alpha, \omega) & =\sup _{\delta>0} \operatorname{lh} a_{\tau}(\alpha, \omega ; \delta), \\
\operatorname{lh} a_{\tau}(\alpha) & =\sup \left\{\operatorname{lh} a_{\tau}(\alpha, \omega) \mid \omega \in \mathscr{P} f(M)\right\} .
\end{aligned}
$$

We have

$$
H(A, \alpha) \leqq \operatorname{lh} a_{\tau}(\alpha, \omega)
$$

when $\omega$ generates $A$ and

$$
H(\alpha) \leqq \operatorname{lh} a_{\tau}(\alpha)
$$

We don't know whether $\operatorname{lh} a_{\tau}(\alpha, \omega), \operatorname{lh} a_{\tau}(\alpha)$ are not actually equal to $h a_{\tau}(\alpha, \omega)$, $h a_{\tau}(\alpha)$. 
1.7. Proposition. Assume $M=L^{\infty}(X, \mathscr{B}, \mu)$ is separable and $\alpha$ is induced by an ergodic measure-preserving automorphism $T$ of the probability measure space $(X, \mathscr{B}, \mu)$ and $\tau$ is the trace defined by $\mu$. Then

$$
h(T)=h a_{\tau}(\alpha)
$$

where $h(T)$ is the Kolmogorov entropy of $T$.

Proof. Since $h(T)=H(\alpha)$ we have $h(T) \leqq h a_{\tau}(\alpha)$ by 1.5 . In view of 1.4 to prove the converse it suffices to show that if $\Omega=\left(\Omega_{1}, \ldots, \Omega_{m}\right)$ is a measurable partition of $X$ and $\omega=\left\{\chi_{\Omega_{j}} \mid \leqq j \leqq m\right\}$, where $\chi_{\Omega_{j}}$ is the indicator function of $\Omega_{j}$, then $h a_{\tau}(\alpha, \omega) \leqq h(T)$. In view of McMillan's theorem, given $\varepsilon>0$ there is $N$ such that, if $n \geqq N$, then in

$$
\Omega^{(n)}=\Omega \vee T \Omega \vee \cdots \vee T^{n-1} \Omega
$$

except for the atoms contained in the set $Z$ with $\mu(Z)<\varepsilon$, the other atoms have measure in the internal $[\exp (-n(h(T)+\varepsilon)), \exp (-n(h(T)-\varepsilon)))$. We then define $A_{n}$ to be the linear span of the $\chi_{\Delta}$, where $\Delta$ ranges over the atoms of $\Omega^{(n)}$ which do not intersect $Z$, plus the set $Z$ itself. Thus $A_{n} \in \mathscr{F}(M)$ and

$$
\operatorname{dim} A_{n} \leqq 1+\exp (n(h(T)+\varepsilon)) .
$$

Since $\left|\chi_{Z}\right|_{2}<\varepsilon^{1 / 2}$ we easily infer

$$
\alpha^{j}(\omega) \subset_{\varepsilon^{1 / 2}} A_{n}
$$

for $0 \leqq j \leqq n-1$. This gives

$$
r_{\tau}\left(\omega \cup \cdots \cup \alpha^{n-1}(\omega) ; \varepsilon^{1 / 2}\right) \leqq 1+\exp (n(h(T)+\varepsilon)),
$$

and hence

$$
h a_{\tau}\left(\alpha, \omega ; \varepsilon^{1 / 2}\right) \leqq h(T)+\varepsilon .
$$

Since $\varepsilon>0$ is arbitrary, this gives the desired conclusion.

1.8. Proposition. Let $M_{k}$ be the algebra of $k \times k$ matrices with its unique tracestate, $M=M_{k}^{\otimes Z}, \tau=\tau_{k}^{\otimes Z}$ and let $\alpha$ be the non-commutative Bernoulli shift on $M$. Then

$$
h a_{\tau}(\alpha)=H(\alpha)=\log k
$$

Proof. Since $h a_{\tau}(\alpha) \geqq H(\alpha)$ by 1.5 we need only prove $h a_{\tau}(\alpha) \leqq H(\alpha)$. Let $A \in$ $\mathscr{F}(M)$ be one of the copies of $\mathscr{M}_{k}$ in the tensor-product defining $M$, and let $\omega$ be a system of matrix units for $A$. By $1.4 h a_{\tau}(\alpha, \omega)=h a_{\tau}(\alpha)$. On the other hand

$$
r_{\tau}\left(\omega \cup \cdots \cup \alpha^{n-1}(\omega) ; \delta\right) \leqq k^{n},
$$

so that $h a_{\tau}(\alpha, \omega) \leqq \log k=H(\alpha)$.

1.9. Proposition. Let $M=M_{1} \otimes M_{2}, \tau=\tau_{1} \otimes \tau_{2}, \alpha=\alpha_{1} \otimes \alpha_{2}$. Then

$$
h a_{\tau_{1} \otimes \tau_{2}}\left(\alpha_{1} \otimes \alpha_{2}\right) \leqq h a_{\tau_{1}}\left(\alpha_{1}\right)+h a_{\tau_{2}}\left(\alpha_{2}\right) \text {. }
$$


Proof. Remark that if $a \in M, B, \in \mathscr{F}(M), b \in B$ are such that $|a-b|_{2}<\delta$, then $b$ may be chosen so that $\|b\| \leqq\|a\|$ (take $b=E_{B} a, E_{B}$ the conditional expectation). If $\omega_{j} \in \mathscr{F}\left(M_{j}\right)$ are such that $x \in \omega_{j} \Rightarrow\|x\|<1$, it is easily seen that

$$
\begin{aligned}
r_{\tau}\left(\bigcup_{0 \leqq J \leqq n-1} \alpha_{1}^{j}\left(\omega_{1}\right) \otimes \alpha_{2}^{j}\left(\omega_{2}\right) ; \delta_{1}+\delta_{2}\right) \leqq & r_{\tau_{1}}\left(\bigcup_{0 \leqq j \leqq n-1} \alpha_{1}^{j}\left(\omega_{1}\right) ; \delta_{1}\right) \\
& +r_{\tau_{2}}\left(\bigcup_{0 \leqq j \leqq n-1} \alpha_{2}^{j}\left(\omega_{2}\right) ; \delta_{2}\right)
\end{aligned}
$$

It follows that

$$
h a_{\tau}\left(\alpha_{1} \otimes \alpha_{2}, \omega_{1} \otimes \omega_{2} ; \delta_{1}+\delta_{2}\right) \leqq h a_{\tau_{1}}\left(\alpha_{1}, \omega_{1} ; \delta_{1}\right)+h a_{\tau_{2}}\left(\alpha_{2}, \omega_{2} ; \delta_{2}\right),
$$

and to get the desired conclusion it suffices to use 1.4.

\section{Subalgebra Approximation the $C^{*}$-Case}

This section runs parallel to the preceding, with hyperfinite $W^{*}$-algebras replaced by $A F C^{*}$-algebras (no state specified). Notations used here do not always have the same meaning as in Sect. 1, being adapted to the AF-case. Thus, $M$ will denote an $A F C^{*}$-algebra with unity. $\mathscr{P} f(M)$ and $\mathscr{F}(M)$ have the same meaning as in Sect. 1. If $\omega \in \mathscr{P} f(M)$ and $X \subset M$ we write $\omega \subset_{\delta} X$ if for every $a \in \omega$ there is $x \in X$ such that $\|a-x\|<\delta$. The definition of the $\delta$-rank $r(\omega ; \delta)$ is a repetition word for word of Definition 1.1, the difference being that $M$ and $\subset_{\delta}$ no longer have the same meaning as in Sect. 1.

2.1. Definition. If $\alpha$ is an automorphism of $M, \omega \in \mathscr{P} f(M)$ and $\delta>0$, we define

$$
\begin{aligned}
\operatorname{hat}(\alpha, \omega ; \delta) & =\limsup _{n \rightarrow \infty} n^{-1} \log r\left(\bigcup_{0 \leqq j \leqq n-1} \alpha^{j}(\omega) ; \delta\right), \\
\operatorname{hat}(\alpha, \omega) & =\sup _{\delta>0} \operatorname{hat}(\alpha, \omega ; \delta), \\
\operatorname{hat}(\alpha) & =\sup \{\operatorname{hat}(\alpha, \omega) \mid \alpha \in \mathscr{P} f(M)\} .
\end{aligned}
$$

hat $(\alpha)$ will be called the topological approximation entropy of $\alpha$.

Clearly the difference between Definition 1.2 and Definition 2.1 is that now $M$ is an $A F$-algebra and $C_{\delta}$ is with respect to the uniform norm.

Propositions 1.3 and 1.4 and their proofs immediately adapt to the context of this section to yield the next two propositions, the proofs of which will be omitted.

2.2 Proposition. If $k \in \mathbb{Z}$ then hat $\left(\alpha^{k}\right)=|k|$ hat $(\alpha)$.

2.3. Proposition. Let $\omega_{j} \in \mathscr{P} f(M), \omega_{1} \subset \omega_{2} \subset \ldots$ be such that $\bigcup_{j \in \mathbb{N}} \bigcup_{n \in \mathbb{Z}}$ $\alpha^{n}\left(\omega_{j}\right)$ generates $M$ as a $C^{*}$-algebra. Then

$$
\operatorname{hat}(\alpha)=\sup _{j \in \mathbb{N}} \operatorname{hat}\left(\alpha, \omega_{j}\right) .
$$


2.4. Proposition. Let $\tau$ be a trace-state of the AF-algebra M such that $\tau \circ \alpha=\tau$. Let $M$ be the von Neumann algebra completion of $M$ with respect to $\tau, \tilde{\alpha}$ the automorphism and $\tilde{\tau}$ the trace obtained from $\alpha$ and $\tau$. Then

$$
h a_{\tilde{\tau}}(\tilde{\alpha}) \leqq h a t(\alpha) .
$$

Proof. Let $\pi: M \rightarrow \tilde{M}$ be the canonical homomorphism. Clearly if $\omega \in \mathscr{P} f(M), A \in$ $\mathscr{F}(M), \omega \subset_{\delta} A$, then $\pi(\omega) \subset_{\delta} \pi(A)$ which also implies the $\delta$-inclusion of $\pi(\omega)$ into $\pi(A)$ with respect to the 2 -norm defined by $\tilde{\tau}$. It follows that if $\omega \in \mathscr{P} f(M)$,

$$
\begin{aligned}
r\left(\omega \cup \cdots \cup \alpha^{n-1}(\omega) ; \delta\right) & \geqq r_{\tilde{\tau}}\left(\pi(\omega) \cup \cdots \cup \pi\left(\alpha^{n-1}(\omega)\right) ; \delta\right) \\
& =r_{\tilde{\tau}}\left(\pi(\omega) \cup \cdots \cup \tilde{\alpha}^{n-1}(\pi(\omega)) ; \delta\right) .
\end{aligned}
$$

Hence $h a t(\alpha, \omega ; \delta) \geqq h a_{\tilde{\tau}}(\pi(\omega) ; \delta)$ and so $h a t(\alpha, \omega) \geqq h a_{\tilde{\tau}}(\tilde{\alpha}, \pi(\omega))$. Using 1.4 and 2.1 we infer $h a t(\alpha) \geqq h a_{\tilde{\tau}}(\tilde{a})$.

2.5. Proposition. Let $T: X \rightarrow X$ be a homeomorphism of a totally disconnected metric space. Let $M=C(X)$ be the $A F C^{*}$-algebra of continuous functions on $X$ and let $\alpha$ be the automorphism induced by $T$. Then we have

$$
h_{\mathrm{top}}(T)=\operatorname{hat}(\alpha),
$$

where $h_{\mathrm{top}}(T)$ is the topological entropy of $T$.

Proof. The topological entropy of $T$ is the supremum of the entropies of $T$ with respect to all ergodic invariant Borel probability measures $\mu$ on $X$ (see [8], p. 273). By 1.7 , the entropy of $T$ acting on $(X, \mu)$ coincides with $h a_{\mu}$ for the automorphism of $L^{\infty}(X, \mu)$ induced by $T$. Since by 2.4 hat $(\alpha)$ majorizes $h a_{\mu}$ of the automorphism of $L^{\infty}(X, \mu)$, we infer hat $(\alpha) \geqq h_{\text {top }}(T)$.

Let $\Omega=\left(\Omega_{1}, \ldots, \Omega_{m}\right)$ be the partition of $X$ into closed open sets and let $\omega=$ $\left\{\chi_{\Omega_{j}} \mid 1 \leqq j \leqq m\right\}$, where $\chi_{\Omega_{j}}$ is the indicator function of $\Omega_{j}$. In view of 2.3 it will suffice to prove that

$$
h a t(\alpha, \omega) \leqq h_{\text {top }}(T) .
$$

With $N(\cdot)$ denoting the least number of elements of an open subcover of a given open cover of $X$, we have

$$
r\left(\omega \cup \cdots \cup \alpha^{n-1}(\omega) ; \delta\right) \leqq N\left(\Omega \vee \cdots \vee T^{n-1} \Omega\right) .
$$

Hence

$$
h a t(\alpha, \omega ; \delta) \leqq h(\Omega, T) \leqq h_{\text {top }}(T)
$$

(for the definition of $h(\omega, T)$ see [8], p. 264). This implies $h(\alpha, \omega) \leqq h_{\mathrm{top}}(T)$.

2.6. Proposition. Let $\mathscr{M}_{k}$ be the $C^{*}$-algebra of $k \times k$ matrices, let $M=\mathscr{M}_{k}^{\otimes \mathbb{Z}}$ be the AF-algebra and let $\alpha$ be the non-commutative topological Bernoulli shift. Then

$$
\operatorname{hat}(\alpha)=\log k .
$$

Proof. Applying 2.4 and 1.8 to the von Neumann algebra completion of $M$ with respect to the unique trace state of $M$ we get $h a t(\alpha) \geqq \log k$. 
On the other hand let $A \in \mathscr{F}(M)$ be one of the copies of $\mathscr{M}_{k}$ in the tensor product defining $M$. Then if $\omega$ is a set of matrix units for $A$, by 2.3 we have hat $(\alpha, \omega)=$ hat $(\alpha)$. On the other hand, since the $C^{*}$-algebra generated by $\omega \cup \cdots \cup$ $\alpha^{n-1}(\omega)$ has rank $k^{n}$, we infer

$$
r\left(\omega \cup \cdots \cup \alpha^{n-1}(\omega) ; \delta\right) \leqq k^{n},
$$

and hence

$$
\operatorname{hat}(\alpha, \omega) \leqq \log k
$$

The proof of the next proposition is entirely analogous to the proof of Proposition 1.9 and will be omitted.

2.7. Proposition. Let $M_{1}, M_{2}$ be AF-algebras, $M=M_{1} \otimes M_{2}, \alpha=\alpha_{1} \otimes \alpha_{2}$. Then

$$
\operatorname{hat}\left(\alpha_{1} \otimes \alpha_{2}\right) \leqq \operatorname{hat}\left(\alpha_{1}\right)+\operatorname{hat}\left(\alpha_{2}\right) .
$$

\section{Completely Positive Approximation the $W^{*}$-Case}

In this section we will work with a weaker type of approximation based on completely positive maps instead of subalgebras. We shall examine only the $W^{*}$-case here and leave the $C^{*}$-case for the next section.

Throughout this section $(M, \sigma)$ is a hyperfinite von Neumann algebra $M$ with a normal faithful state $\sigma$. We assume $\alpha: M \rightarrow M$ is a automorphism so that $\sigma \circ \alpha=\sigma$. Also we shall use the notation $\|a\|_{\sigma}=\left(\sigma\left(a^{*} a\right)\right)^{1 / 2}$, where $a \in M$.

By $C P A(M, \sigma)$ we denote the set of triples $(\varphi, \psi, B)$, where $B$ is a finitedimensional $C^{*}$-algebra, $\varphi: M \rightarrow B$ and $\psi: B \rightarrow M$ are unital completely positive maps so that $\sigma \circ \psi \circ \varphi=\sigma$. If $(\varphi . \psi, B) \in C P A(M, \sigma)$, then $\left(\varphi \circ \alpha^{-n}, \alpha^{n} \circ \psi, B\right) \in$ $C P A(M, \sigma)$.

3.1. Definition. If $\omega \in \mathscr{P} f(M)$ and $\delta>0$ the completely positive $\delta$-rank is defined by

$r c p_{\sigma}(\omega ; \delta)=\inf \left\{\operatorname{rank} B \mid(\varphi, \psi, B) \in C P A(M, \sigma),\|(\psi \circ \varphi)(a)-a\|_{\sigma}<\delta\right.$ for $\left.a \in \omega\right\}$.

Remark that $r c p_{\sigma}\left(\alpha^{n}(\omega) ; \delta\right)=r c p_{\sigma}(\omega ; \delta)$.

3.2. Definition. We define for $\omega \in \mathscr{P} f(M)$ and $\delta>0$,

$$
\begin{aligned}
h c p a_{\sigma}(\alpha, \omega ; \delta) & =\limsup _{n \rightarrow \infty} n^{-1} \log r c p_{\sigma}\left(\omega \cup \alpha(\omega) \cup \cdots \cup \alpha^{n-1}(\omega) ; \delta\right), \\
h c p a_{\sigma}(\alpha, \omega) & =\sup _{\delta>0} h c p a_{\sigma}(\alpha, \omega ; \delta),
\end{aligned}
$$

and

$$
h c p a_{\sigma}(\alpha)=\sup _{\omega \in \mathscr{P} f(M)} h c p a_{\sigma}(\alpha, \omega) .
$$

$h c p a_{\sigma}(\alpha)$ will be called the completely positive approximation entropy. 


\subsection{Proposition.}

$$
h c p a_{\sigma}\left(\alpha^{k}\right)=|k| h c p a_{\sigma}(\alpha) \text {. }
$$

The proof is along the same lines as the proof of 1.3.

3.4. Proposition. Let $\omega_{j} \in \mathscr{P} f(M), \omega_{1} \subset \omega_{2} \subset \ldots$ be such that the linear span of $\bigcup_{\substack{j \in \mathbb{N} \\ k \in \mathbb{Z}}} \alpha^{k}\left(\omega_{j}\right)$ is ultrastrongly dense in $M$. Then

$$
\sup _{j \in \mathbb{N}} h c p a_{\sigma}\left(\alpha, \omega_{j}\right)=h c p a_{\sigma}(\alpha) \text {. }
$$

Proof. Let $\omega \in \mathscr{P} f(M), \omega=\left\{a_{1}, \ldots, a_{m}\right\}$. In view of the assumptions there is $\omega_{N}$ and $p \in \mathbb{N}$ so that if $\bigcup_{-p \leqq k \leqq p} \alpha^{k}\left(\omega_{N}\right)=\left\{x_{1}, \ldots, x_{n}\right\}$, then

$$
\left\|a_{j}-\sum_{1 \leqq k \leqq n} \lambda_{j k} x_{k}\right\|_{\sigma}<\delta
$$

for some scalars $\lambda_{j k}$. With $C=\max _{j, k}\left|\lambda_{j k}\right|$ it is easily seen that

$$
\begin{aligned}
r c p_{\sigma}\left(\omega \cup \cdots \cup \alpha^{d}(\omega) ; 2 \delta\right) & \leqq r c p_{\sigma}\left(\bigcup_{-p \leqq s \leqq d+p} \alpha^{s}\left(\omega_{N}\right) ; C^{-1} \delta\right) \\
& =r c p_{\sigma}\left(\omega_{N} \cup \cdots \cup \alpha^{d+2 p}\left(\omega_{N}\right) ; C^{-1} \delta\right) .
\end{aligned}
$$

This in turn implies

$$
\begin{aligned}
h c p a_{\sigma}(\alpha, \omega ; 2 \delta) & \leqq h c p a_{\sigma}\left(\alpha, \omega_{N} ; C^{-1} \delta\right) \\
& \leqq \sup _{N} h c p a_{\sigma}\left(\alpha, \omega_{N}\right)
\end{aligned}
$$

and hence

$$
h c p a_{\sigma}(\alpha) \leqq \sup _{N} h c p a_{\sigma}\left(\sigma, \omega_{N}\right) .
$$

The opposite inequality is obvious.

3.5. Proposition. Let $N \subset M, 1 \in N$ be a von Neumann subalgebra. Assume $\alpha(N)$ $=N$ and the existence of a projection of norm one $E: M \rightarrow N$ such that $(\sigma \mid N) \circ$ $E=\sigma$. Then

$$
h c p a_{\sigma}(\alpha \mid N) \leqq h c p a_{\sigma}(\alpha) .
$$

Proof. The proposition follows immediately from the fact that $r c p_{\sigma}(\omega ; \delta)$ for a subset $\omega \subset \mathscr{P} f(N)$ is the same w.r.t. $(M, \sigma)$ or $(N, \sigma \mid N)$. This in turn follows from the fact that if $(\varphi, \psi, B) \in C P A(M, \sigma)$, then $(\varphi \mid N, E \circ \psi, B) \in C P A(N, \sigma \mid N)$ and if $a \in N$, then

$$
\begin{aligned}
\|(\psi \circ \varphi)(a)-a\|_{\sigma} & \geqq\|E((\psi \circ \varphi)(a)-a)\|_{\sigma} \\
& =\|(E \circ \psi)(\varphi \mid N)(a)-a\|_{\sigma} .
\end{aligned}
$$

Here we used for $x \in M$

$$
\sigma\left(x^{*} x\right)=\sigma\left(E\left(x^{*} x\right)\right) \geqq \sigma\left(E\left(x^{*}\right) E(x)\right) .
$$


The next proposition related $h c p a_{\sigma}(\alpha)$ with the Connes-Narnhofer-Thirring entropy $h_{\sigma}(\alpha)[3]$. In the proof we will frequently use results and notations from [3].

3.6. Proposition. If $\gamma: A \rightarrow M$ is a completely positive map, A a finite-dimensional $C^{*}$-algebra and $\omega \subset \mathscr{P} f(M)$, so that $\gamma(A)$ is contained in the linear span of $\omega$. Then

$$
h_{\sigma, \alpha}(\gamma) \leqq h c p a_{\sigma}(\alpha, \omega)
$$

and $h_{\sigma}(\alpha) \leqq h c p a_{\sigma}(\alpha)$.

Proof. Let $C>0$ be such that

$$
C^{-1} \gamma(\{a \in A \mid\|a\| \leqq 1\}) \subset\left\{\sum_{x \in \omega} \lambda(x) x\left|\sum_{x \in \omega}\right| \lambda(x) \mid \leqq 1\right\} .
$$

Let $(\varphi, \psi, B) \in C P A(M, \sigma)$ be such that

$$
\|(\psi \circ \varphi)(a)-a\|_{\sigma}<\delta
$$

for $a \in \omega \cup \alpha(\omega) \cup \cdots \cup \alpha^{n-1}(\omega)$. Then

$$
\left\|\left((\psi \circ \varphi) \circ \alpha^{j} \circ \gamma\right)(a)-\left(\alpha^{j} \circ \gamma\right)(a)\right\|_{\sigma}<C \delta
$$

if $a \in A,\|a\| \leqq 1$ and $0 \leqq j \leqq n-1$. Given $\varepsilon>0$ by theorem VI.3 in [3] there is a corresponding $\delta>0$ such that this implies (for all $n$ ):

$$
\left|H_{\sigma}\left(\gamma, \ldots, \alpha^{n-1} \circ \gamma\right)-H_{\sigma}\left(\psi \circ \varphi \circ \gamma, \ldots, \psi \circ \varphi \circ \alpha^{n-1} \circ \gamma\right)\right|<n \varepsilon .
$$

Using Proposition III.6 and the observation following Definition III.4 in [3] we have

$$
H_{\sigma}\left(\left(\psi \circ \varphi \circ \alpha^{j} \circ \gamma\right)_{0 \leqq j \leqq n-1}\right) \leqq H_{\sigma}(\psi) \leqq S(\sigma \circ \psi) \leqq \log \operatorname{rank} B .
$$

Since we may choose

$$
\operatorname{rank} B=r c p_{\sigma}\left(\omega \cup \cdots \cup \alpha^{n-1}(\omega) ; \delta\right),
$$

we infer

$$
\begin{aligned}
h_{\sigma, \alpha}(\gamma) & \leqq h c p a_{\sigma}(\alpha, \omega ; \delta)+\varepsilon \\
& \leqq h c p a_{\sigma}(\alpha, \omega)+\varepsilon .
\end{aligned}
$$

Since $\varepsilon>0$ is arbitrary we get $h_{\sigma, \alpha}(\gamma) \leqq h c p a_{\sigma}(\alpha, \omega)$. The inequality $h_{\sigma}(\alpha) \leqq$ $h \operatorname{cpa}_{\sigma}(\alpha)$ is an obvious consequence.

3.7. Proposition. Assume $\sigma=\tau$ is a faithful normal trace-state. Then

$$
h c p a_{\tau}(\alpha) \leqq h a_{\tau}(\alpha) \text {. }
$$

Proof. The proposition follows easily from the following remark. If $B \in \mathscr{F}(M)$ is unital, let $i_{B}: B \rightarrow M$ be the inclusion and $E_{B}: M \rightarrow B$ the conditional expectation given by the projection in $L^{2}(M, \tau)$. Then

$$
\left(E_{B}, i_{B}, B\right) \in C P A(M, \tau) .
$$


Combining 1.7, 3.6 and 3.7 we obtain the following corollary.

3.8. Corollary. If $M$ is commutative and separable and $\alpha$ is ergodic then

$$
h(\alpha)=h c p a_{\tau}(\alpha) \text {. }
$$

3.9. Proposition. Let $\sigma_{k}$ be a faithful state on $\mathscr{M}_{k}$. Let $\sigma=\sigma_{k}^{\otimes \mathbb{Z}}$ on $M=\mathscr{M}_{k}^{\otimes \mathbb{Z}}$ and let $\alpha$ be the non-commutative Bernoulli shift. Then

$$
h_{\sigma}(\alpha)=h c p a_{\sigma}(\alpha) \text {. }
$$

Proof. Let $D_{k} \subset \mathscr{M}_{k}$ be a m.a.s.a. such that $\sigma_{k}=\operatorname{Tr}(\cdot X)$ for some $X \in D_{k}$ and let $D=D_{k}^{\otimes \mathbb{Z}} \subset M$. By the results of [3] we have

$$
h_{\sigma}(\alpha)=h_{\sigma \mid D}(\alpha \mid D) \text {. }
$$

Let further $M^{(n)}=\mathscr{M}_{k}^{\otimes[0, n]} \subset M$ and $D^{(n)}=M^{(n)} \cap D$. By McMillan's theorem there are self-adjoint projections $P_{n} \in D^{(n)}$ such that $\sigma\left(P_{n}\right) \rightarrow 1$ as $n \rightarrow \infty$ and $n^{-1} \log$ rank $\left(P_{n} D^{(n)}\right)-h_{\sigma}(\alpha) \rightarrow 0$. Let $B_{n}=\left(I-P_{n}\right)+P_{n} M^{(n)} P_{n}$. Choose finite subsets $\omega_{n} \subset M^{(n)}$ which span $M^{(n)}$ such that $j<k \Rightarrow \omega_{j} \subset \omega_{k}$. By $3.4 h c p a_{\sigma}(\alpha)=$ $\sup _{n} h c p a_{\sigma}\left(\alpha, \omega_{n}\right)$. Let $E_{n}$ be the conditional expectation onto $M^{(n)}$ given by $\left(\mathrm{id}_{M_{k}}^{\otimes[0, n]}\right.$ $\otimes \sigma_{k}^{\otimes(\mathbb{Z} \backslash[0, n])}$, and let

$$
\varphi_{n}(a)=P_{n} E_{n}(a) P_{n}+\sigma\left(\left(I-P_{n}\right) a\left(I-P_{n}\right)\right) \cdot\left(\sigma\left(I-P_{n}\right)\right)^{-1}\left(I-P_{n}\right) .
$$

It is easily seen that $\sigma \circ \varphi_{n}=\sigma$ and

$$
\lim _{n \rightarrow \infty}\left(\sup \left\{\left\|\varphi_{n+p}(a)-a\right\|_{\sigma} \mid a \in \bigcup_{0 \leqq j \leqq n} \alpha^{j}\left(\omega_{p}\right)\right\}\right)=0
$$

(note that $P_{n+p} \varphi_{n+p}(a)=P_{n+p} a P_{n+p}$, for $a$ as in the last formula). This implies

$$
h c p a_{\sigma}\left(\alpha, \omega_{p}\right) \leqq \limsup _{n \rightarrow \infty} n^{-1} \log \operatorname{rank} B_{n}=h_{\sigma}(\alpha),
$$

which gives

$$
h c p a_{\sigma}(\alpha) \leqq h_{\sigma}(\alpha) \text {. }
$$

The opposite inequality was already obtained in general in 3.6.

3.10. Proposition. Let $M=M_{1} \otimes M_{2}, \sigma=\sigma_{1} \otimes \sigma_{2}, \alpha=\alpha_{1} \otimes \alpha_{2}$. Then

$$
\begin{aligned}
& h c p a_{\sigma_{1} \otimes \sigma_{2}}\left(\alpha_{1} \otimes \alpha_{2}\right) \leqq h c p a_{\sigma 1}\left(\alpha_{1}\right)+h c p a_{\sigma 2}\left(\alpha_{2}\right), \\
& h c p a_{\sigma_{1} \otimes \sigma_{2}}\left(\alpha_{1} \otimes \alpha_{2}\right) \geqq \max \left(h c p a_{\sigma 1}\left(\alpha_{1}\right), h c p a_{\sigma 2}\left(\alpha_{2}\right)\right) .
\end{aligned}
$$

Proof. If $a_{j} \in M_{j},\left(\varphi_{j}, \psi_{j} ; B_{j}\right) \in C P A\left(M_{j}, \sigma_{j}\right)$, then

$$
\begin{aligned}
\left\|\left(\left(\psi_{1} \otimes \psi_{2}\right) \circ\left(\varphi_{1} \otimes \varphi_{2}\right)\right)\left(a_{1} \otimes A_{2}\right)-a_{1} \otimes a_{2}\right\|_{\sigma} \leqq & \left\|\left(\psi_{1} \circ \varphi_{1}\right)\left(a_{1}\right)-a_{1}\right\|_{\sigma_{1}}\left\|a_{2}\right\| \\
& +\left\|a_{1}\right\|\left\|\left(\psi_{2} \circ \varphi_{2}\right)\left(a_{2}\right)-a_{2}\right\|_{\sigma} .
\end{aligned}
$$

Thus if $\omega_{j} \in \mathscr{P} f\left(M_{j}\right)$ are such that $x \in \omega_{j} \Rightarrow\|x\|<1$, we have 


$$
\begin{aligned}
r c p_{\sigma_{1} \otimes \sigma_{2}}\left(\bigcup_{0 \leqq J \leqq n-1}\left(\alpha_{1} \otimes \alpha_{2}\right)^{j}\left(\omega_{1} \otimes \omega_{2}\right) ; \delta_{1}+\delta_{2}\right) \leqq & r c p_{\sigma_{1}}\left(\bigcup_{0 \leqq J \leqq n-1} \alpha_{1}^{j}\left(\omega_{1}\right) ; \delta_{1}\right) \\
& +r c p_{\sigma_{2}}\left(\bigcup_{0 \leqq J \leqq n-1} \alpha_{2}^{j}\left(\omega_{2}\right) ; \delta_{2}\right) .
\end{aligned}
$$

This in turn gives

$$
h c p a_{\sigma_{1} \otimes \sigma_{2}}\left(\alpha_{1} \otimes \alpha_{2}, \omega_{1} \otimes \omega_{2} ; \delta_{1}+\delta_{2}\right) \leqq h c p a_{\sigma_{1}}\left(\alpha_{1}, \omega_{1} ; \delta_{1}\right)+h c p a_{\sigma_{2}}\left(\alpha_{2}, \omega_{2} ; \delta_{2}\right),
$$

and using 3.4 we get the desired conclusion.

The second inequality follows immediately from Proposition 3.5.

3.11. Let $\alpha$ be a Power's shift, i.e. assume $M$ is generated by $e_{j}(j \in \mathbb{Z}), e_{j}=$ $e_{j}^{*}, e_{j}^{2}=1, e_{p} e_{q}=(-1)^{\gamma(|p-q|)} e_{q} e_{p}$ for some function $\gamma: \mathbb{N} \rightarrow\{0,1\}$ and $\sigma=\tau$ is a faithful trace-state so that $\tau\left(e_{j}\right)=0$ and $\alpha\left(e_{j}\right)=e_{j+1}$.

Proposition. If $\alpha$ is a Power's shift on $(M, \tau)$, then

$$
2^{-1} \log 2 \leqq h c p_{\tau}(\alpha) \leqq h a_{\tau}(\alpha) \leqq \log 2
$$

Proof. Since the algebra generated by $e_{0}, e_{1}, \ldots, e_{n-1}$ has dimension $2^{n}$ we easily get $h a_{\tau}(\alpha) \leqq \log 2$.

On the other hand let $N \subset M$ be the subalgebra generated by $e_{j} \otimes e_{j}$.

Then $(\alpha \otimes \alpha) \mid N$ is a classical Bernoulli shift and $h((\alpha \otimes \alpha) \mid N)=\log 2$. Thus $h c p_{\tau}(\alpha) \geqq 2^{-1} h c p_{\tau}(\alpha \otimes \alpha) \geqq 2^{-1} h c p_{\tau}((\alpha \otimes \alpha) \mid N)=2^{-1} \log 2$.

In [15] the existence of Power's shifts for which $h(\alpha)=0$ is proved and therefore $h(\alpha) \neq h c p_{\tau}(\alpha)$.

\section{Topological Entropy (Completely Positive Approximation the $C^{*}$-Case)}

This section is the $C^{*}$-parallel to Sect. 3. The dynamical invariant we obtain will be the topological entropy.

Here $M$ will be a nuclear $C^{*}$-algebra with unity and $\alpha$ an automorphism. By $C P A(M)$ we denote triples $(\varphi, \psi, B)$, where $B$ is a finite-dimensional $C^{*}$-algebra and $\varphi: M \rightarrow B, \psi: B \rightarrow M$ are unital completely positive maps. The completely positive $\delta$-rank is then defined by

$$
\begin{aligned}
\operatorname{rcp}(\omega ; \delta)= & \inf \{\operatorname{rank} B \mid(\varphi, \psi, B) \in C P A(M),\|(\psi \circ \varphi)(a)-a\| \\
& <\delta \text { for } a \in \omega\} .
\end{aligned}
$$

\subsection{Definition.}

$$
\begin{aligned}
h t(\alpha, \omega ; \delta) & =\limsup _{n \rightarrow \infty} n^{-1} \log r c p\left(\omega \cup \alpha(\omega) \cup \cdots \cup \alpha^{n-1}(\omega) ; \delta\right), \\
h t(\alpha, \omega) & =\sup _{\delta>0} h t(\alpha, \omega ; \delta), \\
h t(\alpha) & =\sup _{\omega \in \mathscr{P} f(M)} h t(\alpha, \omega) .
\end{aligned}
$$

$h t(\alpha)$ is called the topological entropy of $\alpha$. 
The following two propositions and their proofs are the obvious analogues of Propositions 3.3 and 3.4 , the proofs are left to the reader.

\subsection{Proposition.}

$$
h t\left(\alpha^{k}\right)=|k| h t(\alpha) .
$$

4.3. Proposition. Let $\omega_{j} \in \mathscr{P} f(M), \omega_{1} \subset \omega_{2} \subset \ldots$ be such that the linear span of $\bigcup_{\substack{j \in \mathbb{Z} \\ k \in \mathbb{Z}}} \alpha^{k}\left(\omega_{j}\right)$ is dense on $M$. Then

$$
\sup _{j \in \mathbb{N}} h t\left(\alpha, \omega_{j}\right)=h t(\alpha)
$$

We also have the analogue of Proposition 3.5.

4.4 Proposition. Let $1 \in N \subset M$ be a $C^{*}$-subalgebra and assume there is a projection of norm one $E$ of $M$ onto $N$. Then

$$
h t(\alpha \mid N) \leqq h t(\alpha) .
$$

The proof is along the same lines as that of 3.5 and will be omitted. As a biproduct one has that for $\omega \in \mathscr{P} f(N), r c p(\omega ; \delta)$ is the same w.r.t. $N$ or $M$.

4.5. Proposition. Assume $M$ is an AF-algebra. Then

$$
h t(\alpha) \leqq h a t(\alpha) .
$$

Proof. Let $\omega \in \mathscr{P} f(M)$ and let $B_{n} \in \mathscr{F}(M)$ be such that

$$
\omega \cup \cdots \cup \alpha^{n-1}(\omega) \subset_{\delta} B_{n}
$$

and $\lim \sup _{n \rightarrow \infty} n^{-1} \log$ rank $B_{n} \leqq$ hat $\alpha$.

Since $B_{n}$ is a finite-dimensional $C^{*}$-subalgebra, there is a projection of norm one $E_{n}: M \rightarrow B_{n}$. Denoting by $i_{n}: B_{n} \rightarrow M$ the inclusion, we have

$$
\left\|\left(i_{n} \circ E_{n}\right)(a)-a\right\|<2 \delta,
$$

if $a \in \omega \cup \cdots \cup \alpha^{n-1}(\omega)$ and hence $h t(\alpha, \omega ; 2 \delta) \leqq$ hat $\alpha$.

Since $\delta>0$ is arbitrary we get

$$
h t(\alpha, \omega) \leqq h a t(\alpha),
$$

and hence $h t(\alpha) \leqq h a t(\alpha)$.

4.6. Proposition. Let $\sigma$ be an $\alpha$-invariant state on $M$. Then

$$
h_{\sigma}(\alpha) \leqq h t(\alpha)
$$

Proof. The proof is similar to that of Proposition 3.6. Let $\gamma: A \rightarrow M$ be a unital completely positive map and let $\omega \in \mathscr{P} f(M)$ be such that $\gamma(\{a \in A \mid\|a\| \leqq 1\})$ is contained in the convex hull of $\omega$. Let $(\varphi, \psi, B) \in C P A(M)$ be such that

$$
\|(\psi \circ \varphi)(a)-a\|<\delta
$$


for $a \in \omega \cup \alpha(\omega) \cup \cdots \cup \alpha^{n-1}(\omega)$. Then if $a \in A,\|a\| \leqq 1$ and $0 \leqq j \leq n-1$,

$$
\left\|\left((\psi \circ \varphi) \circ \alpha^{j} \circ \gamma\right)(a)-\left(\alpha^{j} \circ \gamma\right)(a)\right\|<\delta .
$$

By Proposition IV.3 in [3], given $\varepsilon>0$, there is a corresponding $\delta>0$, such that the previous inequality implies (for all $n$ )

$$
\mid H_{\sigma}\left(\left(\alpha^{j} \circ \gamma\right)_{0 \leqq j \leqq n-1}-H_{\sigma}\left(\left(\psi \circ \varphi \circ \alpha^{j} \circ \gamma\right)_{0 \leqq j \leqq n-1}\right) \mid<n \varepsilon .\right.
$$

Using Proposition III.6 a) and c) and the observation after Definition III.4 in [3], we have

$$
\begin{aligned}
H_{\sigma}\left(\left(\psi \circ \varphi \circ \alpha^{j} \circ \gamma\right)_{0 \leqq j \leqq n-1}\right. & \leqq H_{\sigma}(\psi) \\
& \leqq S(\sigma \circ \psi) \leqq \log \operatorname{rank} B .
\end{aligned}
$$

Like in the proof of Proposition 3.6 this then leads to

$$
h_{\sigma, \alpha}(\gamma) \leqq h t(\alpha, \omega)
$$

and $h_{\sigma}(\alpha) \leqq h t(\alpha)$.

4.7. Proposition. Let $M=\mathscr{M}_{k}^{\otimes \mathbb{Z}}$ and let $\alpha$ be the non-commutative topological Bernoulli shift. Then

$$
h t(\alpha)=\log k
$$

Proof. By 4.5 and 2.6

$$
h t(\alpha) \leqq h a t(\alpha)=\log k
$$

On the other hand let $\tau$ be the unique trace-state on $M$, then by 4.6 ,

$$
h t(\alpha) \geqq h_{r}(\alpha)=\log k .
$$

4.8. Proposition. Let $T: X \rightarrow X$ be a homeomorphism of a compact metric space. Let $M=C(X)$ be the $C^{*}$-algebra of continuous functions on $X$ and let $\alpha$ be the automorphism induced by $T$. Then

$$
h_{\text {top }}(T)=h t(\alpha) \text {. }
$$

Proof. By $4.6 h t(\alpha)$ is $\geqq$ the supremum of $h_{\sigma}(\alpha)$, where $\sigma$ runs over the $\alpha$-invariant states of $C(X)$ and thus $h t(\alpha) \geqq h_{\text {top }}(T)$. For the converse we shall use the notations and definitions on 264 and 265 of [8]. Let $\omega \in \mathscr{P} f(C(X))$ and $\mathscr{U}=\left\{U_{1}, \ldots, U_{m}\right\}$ be an open cover of $X$ such that $x, y \in U_{j} \Rightarrow|f(x)-f(y)|<\delta$ for all $f \in \omega, 1 \leqq$ $j \leqq n$. Let $\mathscr{V}$ be a subcover of $\mathscr{U}_{0}^{n-1}=\bigvee_{0 \leqq j \leqq n-1} T^{j} \mathscr{U}$ with minimal number $N\left(\mathscr{U}_{0}^{n-1}\right)$ of elements. Then if $f \in \bigcup_{0 \leqq j \leqq n-1} \alpha^{j}(\omega)$ and $V \in \mathscr{V}$ we have $x, y \in$ $V \Rightarrow|f(x)-f(y)|<\delta$. Let $\mathscr{V}=\left\{V_{j} \mid 1 \leqq j \leqq N\left(\mathscr{U}_{0}^{n-1}\right)\right\}$ and let $x_{j} \in V_{j}, X_{n}=$ $\left\{x_{j} \mid 1 \leqq j \leqq N\left(\mathscr{U}_{0}^{n-1}\right)\right\}$ and $\chi_{j} \in C(X), 1 \leqq j \leqq N\left(\mathscr{U}_{0}^{n-1}\right), 0 \leqq \chi_{j} \leqq 1$, supp $\chi_{j} \subset$ $V_{j}, \sum_{j} \chi_{j}=1$ a partition of unity subordinate to $\mathscr{V}$. We define

$$
B_{n}=C\left(X_{n}\right), \varphi_{n}: M \rightarrow B_{n}
$$


by

$$
C(X) \ni f \rightarrow f \mid X_{n} \in C\left(X_{n}\right)
$$

and $\psi_{n}: B_{n} \rightarrow M$ by

$$
C\left(X_{n}\right) \ni g \rightarrow \sum_{1 \leqq j \leqq N\left(\|_{0}^{n-1}\right)} g\left(x_{j}\right) \chi_{j} \in C(X) .
$$

If $f \in \bigcup_{0 \leqq j \leqq n-1} \alpha^{j}(\omega)$, then

$$
\begin{aligned}
\left|f(x)-\left(\left(\psi_{n} \circ \varphi_{n}\right)(f)\right)(x)\right| & \leqq \sum_{j} \chi_{j}(x)\left|f(x)-f\left(x_{j}\right)\right| \\
& =\sum_{\left\{j \mid x \in V_{J}\right\}} \chi_{j}(x)\left|f(x)-f\left(x_{j}\right)\right|<\delta .
\end{aligned}
$$

Thus

$$
r c p\left(\bigcup_{0 \leqq j \leqq n-1} \alpha^{j}(\omega) ; \delta\right) \leqq \operatorname{rank} B_{n}=N\left(\mathscr{U}_{0}^{n-1}\right)
$$

and hence

$$
h t(\alpha, \omega ; \delta) \leqq h(\mathscr{U}, T) \leqq h_{\text {top }}(T) .
$$

This in turn implies $h t(\alpha) \leqq h_{\text {top }}(T)$.

The proof of the next proposition is similar to the proof of Proposition 3.10 and will be omitted.

4.9. Proposition. Let $M=M_{1} \otimes M_{2}, \alpha=\alpha_{1} \otimes \alpha_{2}$. Then

$$
h t\left(\alpha_{1} \otimes \alpha_{2}\right) \leqq h t\left(\alpha_{1}\right)+h t\left(\alpha_{2}\right)
$$

and

$$
h t\left(\alpha_{1} \otimes \alpha_{2}\right) \geqq \max \left(h t\left(\alpha_{1}\right), h t\left(\alpha_{2}\right)\right)
$$

\section{Automorphisms of Non-commutative Tori}

This section deals with automorphisms of non-commutative tori, the main result being that the topological entropy is $\leqq$ the entropy of the corresponding automorphism of the commutative torus.

Let $A_{\rho}, \rho=\left(\rho_{l j}\right)_{1 \leqq i, j \leqq n}$ be a $n$-dimensional non-commutative torus, i.e. $A_{\rho}$ is a $C^{*}$-algebra generated by unitaries $u_{1}, \ldots, u_{n}$ such that $u_{l} u_{j}=\rho_{i j} u_{j} u_{i}$, where $\left|\rho_{i j}\right|=1$ and there is an action $\beta: \mathbb{T}^{n} \rightarrow \operatorname{Aut}\left(A_{\rho}\right)$ such that $\beta\left(e^{i \theta_{1}}, \ldots, e^{i \theta_{n}}\right)\left(u_{j}\right)=e^{i \theta_{j}} u_{j}$. Let $T \in G L(n, \mathbb{Z})$ be an integral matrix with det $T= \pm 1$ and assume there is an automorphism $\alpha$ of $A$ such that $\alpha\left(u_{j}\right)=u_{1}^{m_{1 j}} \ldots u_{n}^{m_{n j}}$. Let further $\tau$ denote the $\beta$-invariant trace-state on $A$ given by $\tau\left(u_{1}^{k_{1}} \ldots u_{n}^{k_{n}}\right)=\delta_{0, k_{1}} \ldots \delta_{0, k_{n}}$. The representation $\pi$ associated by the $G N S$ construction with $\tau$ is realized in $l^{2}\left(\mathbb{Z}^{n}\right)$, where we identify $e(k), k=\left(k_{1}, \ldots, k_{n}\right) \in \mathbb{Z}^{n}$ with the vector $\pi\left(u^{k}\right) \zeta=\pi\left(u_{1}^{k_{1}} \ldots u_{n}^{k_{n}}\right) \zeta$ ( $\zeta$ the cyclic vector). Let $\mathscr{J}$ be the canonical antiunitary involution $\mathscr{J} \pi(x) \zeta=\pi\left(x^{*}\right) \zeta$.

Given linearly independent vectors $v_{1}, \ldots, v_{n}$ in $\mathbb{R}^{n}$, consider the parallelipiped

$$
P(R)=\left\{\sum \lambda_{j} v_{j}|| \lambda_{j} \mid \leqq R, \lambda_{j} \in \mathbb{R}\right\}
$$


and

$$
Q(R)=P(R) \cap \mathbb{Z}^{n} .
$$

5.1. Lemma. Given $\varepsilon>0$ there is $\lambda>0$, such that if

$$
P(R) \supset \Delta=\left\{h \in \mathbb{R}^{n}|| h_{j} \mid \leqq 1,1 \leqq j \leqq n\right\},
$$

then there are unital completely positive maps

$$
\begin{aligned}
& \varphi: A_{\rho} \rightarrow B\left(l^{2}(Q(\lambda R))\right), \\
& \psi: B\left(l^{2}(Q(\lambda R))\right) \rightarrow A_{\rho},
\end{aligned}
$$

so that

$$
\left\|(\psi \circ \varphi)\left(u^{k}\right)-u^{k}\right\|<\varepsilon
$$

if $k \in Q(R)$.

Proof. Let $F$ be the orthogonal projection of $l^{2}\left(\mathbb{Z}^{n}\right)$ onto $l^{2}(Q(\lambda R))$. We define $\varphi(a)=F \pi(a) F \mid l^{2}(Q(\lambda R))$. We identify $B\left(l^{2}(Q(\lambda R))\right)$ with $F B\left(l^{2}\left(\mathbb{Z}^{n}\right)\right) F$ and we define

$$
\tilde{\psi}: B\left(l^{2}(Q(\lambda R))\right) \rightarrow B\left(l^{2}\left(\mathbb{Z}^{n}\right)\right)
$$

by

$$
\tilde{\psi}(X)=w-\lim _{p \rightarrow \omega}|Q(\lambda R)|^{-1} \sum_{k \in Q(p)} \mathscr{J} \pi\left(u^{k}\right) \mathscr{J} X\left(\mathscr{J} \pi\left(u^{k}\right) \mathscr{J}\right)^{*}
$$

where $\omega$ is a non-trivial ultrafilter on $\mathbb{N}$. Clearly $\varphi$ and $\tilde{\psi}$ are completely positive. Let $E_{k l}\left(k, l \in \mathbb{Z}^{n}\right)$ be the system of matrix units in $B\left(l^{2}\left(\mathbb{Z}^{n}\right)\right)$ and consider also $V_{k l}=\pi\left(u^{k-l}\right) E_{l l}$ so that $V_{k l}=\gamma_{k l} E_{k l},\left|\gamma_{k l}\right|=1$. If $a, b, k \in \mathbb{Z}^{n}$, then

$$
\mathscr{J} \pi\left(u^{k}\right) \mathscr{J} V_{a b}\left(\mathscr{J} \pi\left(u^{k}\right) \mathscr{J}\right)^{*} e_{q}=\delta_{b-k, q} V_{a-k, b-k} e_{q}
$$

Hence, if $a, b \in Q(\lambda R)$, we have

$$
\sum_{k \in Q(p)} \mathscr{J} \pi\left(u^{k}\right) \mathscr{J} V_{a b}\left(\mathscr{J} \pi\left(u^{k}\right) \mathscr{J}\right)^{*} e_{q}=|\{b-q\} \cap Q(p)| \pi\left(u^{a-b}\right) e_{q} .
$$

We infer

$$
\tilde{\psi}\left(V_{a b}\right)=|Q(\lambda R)|^{-1} \pi\left(u^{a-b}\right) .
$$

Since the $V_{a b}$ with $a, b \in Q(\lambda R)$ span $B\left(l^{2}(Q(\lambda R))\right)$, it follows that $\tilde{\psi}=\pi \circ \psi$, where $\psi: B\left(l^{2}(Q(\lambda R))\right) \rightarrow A_{\rho}$ is a completely positive map and $\psi\left(V_{a b}\right)=|Q(\lambda R)|^{-1} u^{a-b}$. This gives

$$
\begin{aligned}
\psi\left(\varphi\left(u^{c}\right)\right) & =\psi\left(F \pi\left(u^{c}\right) F\right) \\
& =\sum_{b \in Q(\lambda R) \cap(Q(\lambda R)-c)} \psi\left(V_{c+b, b}\right) \\
& =|Q(\lambda R)|^{-1}|Q(\lambda R) \cap(Q(\lambda R)-c)| u^{c} .
\end{aligned}
$$

$\varphi$ is clearly unital and the last formula (for $c=0$ ) inplies $\psi$ is unital. We also get that if $c \in Q(R)$ :

$$
\left\|\psi\left(\varphi\left(u^{c}\right)\right)-u^{c}\right\| \leqq 1-\frac{|Q((\lambda-1) R)|}{|Q(\lambda R)|} .
$$


It is easy to see that if $\Delta \subset P(R)$ and $\lambda \geqq 3$ then

$$
|Q(\lambda R)| \leqq \operatorname{vol}(P((\lambda+1) R))
$$

and

$$
|Q((\lambda-1) R)| \geqq \operatorname{vol}(P((\lambda-2) R)) .
$$

Then choosing $\lambda$ large enough, we will have

$$
1-\frac{|Q((\lambda-1) R)|}{|Q(\lambda R)|} \leqq 1-\left(\frac{\lambda-2}{\lambda-1}\right)^{n}<\varepsilon .
$$

5.2. Lemma. Let $T \in G L(n, R)$ and let $\lambda_{1}, \ldots, \lambda_{n}$ be its eigenvalues and $\mu_{j}=$ $\max \left(1,\left|\hat{\lambda}_{j}\right|\right)$. Then there is a basis $v_{1}, \ldots, v_{n}$ of $\mathbb{R}^{n}$ such that if $\varepsilon>0$ and $\sigma \subset \mathbb{R}^{n}$ is a finite subset, then there is $n_{0} \in \mathbb{N}$, so that if $N \geqq n_{0}$, then

$$
\left\{\sum_{1 \leqq j \leqq n} s_{j}(1+\varepsilon)^{N} \mu_{j}^{N} v_{j}|| s_{j} \mid \leqq 1,1 \leqq j \leqq n\right\} \supset\left\{T^{m} h \mid h \in \sigma, 0 \leqq m \leqq N\right\} .
$$

Proof. For each real eignevalue $\lambda$ of $T$ choose a basis in the spectral subspace for $\{\lambda\}$ of $A$ and for each pair of conjugate complex eigenvalues $\lambda, \bar{\lambda}$ of $A$ choose a basis for the spectral subspace of $A$ corresponding to $\{\lambda, \bar{\lambda}\}$ (in $\mathbb{R}^{n}$ ). Putting these together we get the basis $v_{1}, \ldots, v_{n}$ with the required property. The factor $(1+\varepsilon)$ is necessary because Jordan cells may be present.

5.3. Proposition. Let $\alpha$ be an automorphism of the non-commutative torus $A_{\rho}$ corresponding to a matrix $T \in G L(n, \mathbb{Z})$. Then $h t(\alpha) \leqq \log \left(\mu_{1} \mu_{2} \ldots \mu_{n}\right)$, where $\mu_{j}=\max \left(1,\left|\lambda_{j}\right|\right)$ and $\lambda_{1}, \ldots, \lambda_{n}$ are the eigenvalues of $T$ (each repeated according to its spectral multiplicity).

Proof. Given $\delta>0$ and $\varepsilon>0$ it will sufice to show that for $\omega=\left\{u^{k} \mid k \in \sigma\right\} \sigma$ a finite subset of $\mathbb{Z}^{n}$, we have

$$
r c p\left(\omega \cup \alpha(\omega) \cup \cdots \cup \alpha^{N-1}(\omega) ; \delta\right) \leqq C(1+\varepsilon)^{N_{n}}\left(\mu_{1} \ldots \mu_{n}\right)^{N}
$$

for $N \geqq n_{0}$ (some $n_{0} \in \mathbb{N}$ ). Indeed choose vectors $v_{1}, \ldots, v_{n}$ as provided by 5.2 . Let $P_{N}(1)$ be the parallelipiped

$$
\left\{\sum_{1 \leqq 1 \leqq n} s_{j}(1+\varepsilon)^{N} \mu_{j}^{N} v_{j}|| s_{j} \mid \leqq 1,1 \leqq j \leqq n\right\}
$$

spanned by the vectors $(1+\varepsilon)^{N} \mu_{j}^{N} v_{j}, 1 \leqq j \leqq n$. Enlarging the set $\sigma$ so that it contains $\left\{k \in \mathbb{Z}^{n}|| k_{j} \mid \leqq 1,1 \leqq j \leqq n\right\}$ we have that $P_{N}(1)$ satisfies the condition $P_{N}(1) \supset \Delta$ in Lemma 5.1. Hence there is $\lambda>0$ and there are unital completely positive maps

$$
\begin{gathered}
\varphi_{N}: A_{\rho} \rightarrow B_{N} \\
\psi_{N}: B_{N} \rightarrow A_{\rho}
\end{gathered}
$$

with rank $B_{N}=\left|Q_{N}(\lambda)\right|$ satisfying

$$
\left\|\left(\psi_{N} \circ \varphi_{N}\right)\left(u^{k}\right)-u^{k}\right\|<\delta
$$


if $k \in Q_{N}(1)$. (Here $Q_{N}(R)=P_{N}(R) \cap \mathbb{Z}^{n}$ and $B_{N}=B\left(l^{2}\left(Q_{N}(\lambda)\right)\right)$.) Since $\bigcup_{m=0}^{N-1} T^{m}$ $\sigma \subset Q_{N}(R)$, we get

$$
\left\|\left(\psi_{N} \circ \varphi_{N}\right)(x)-x\right\|<\varepsilon
$$

if $x \in \omega \cup \cdots \cup \alpha^{N-1}(\omega)$. We have

$$
\operatorname{rank} B_{N}=\left|Q_{N}(\lambda)\right| \leqq \operatorname{vol}\left(P_{N}(\lambda+1)\right)=C(1+\varepsilon)^{N_{n}}\left(\mu_{1} \ldots \mu_{n}\right)^{N}
$$

for some constant $C>0$.

5.4. Corollary. Let $\alpha$ be an automorphism of the non-commutative torus $A_{\rho}$ corresponding to a matrix $T \in G L(n, \mathbb{Z})$. If $v$ is an $\alpha$-invariant state on $A_{\rho}$ and $\mu_{j}=\max \left(1,\left|\lambda_{j}\right|\right), \lambda_{1}, \ldots, \lambda_{n}$ being the eigenvalues of $T$, then

$$
h_{v}(\alpha) \leqq \log \left(\mu_{1} \mu_{2} \ldots \mu_{n}\right)
$$

Proof. This follows from 4.6 and 5.3.

5.5. Remark. For the particular case of the irrational rotation algebra (i.e. $n=2$ ) endowed with the trace state $\tau$, a proof of $H(\alpha) \leqq \log \mu_{1} \mu_{2}$ is given in [7]. If valid, that proof would imply $h a_{\tau}(\alpha) \leqq \log \mu_{1} \mu_{2}$. Unfortunately important details are missing, which we were unable to fill in (perhaps it may be necessary to assume the irrational number has special diophantine properties).

\section{The Crossed Product of the Bernoulli Shift}

With essentially the same argument used for automorphisms of non-commutative tori we compute here the topological entropy of the inner automorphism implementing the topological Bernoulli shift in its covariance algebra. In particular, this answers in the affirmative for measurable Bernoulli shifts of entropy $\log n$ a question of Störmer [11].

Let $X=\{1, \ldots, n\}^{Z}$ and consider the crossed product $C(X) \triangleleft_{\beta} \mathbb{Z}$, where $\beta$ is the Bernoulli-shift action. We will show $h t(\operatorname{Ad} u), u$ the implementing unitary of $\beta(1)$, coincides with the topological entropy of the Bernoulli shift, i.e. $\log n$. This is equivalent to computing the topological entropy of a certain inner group automorphiism.

We begin with a construction similar to the one in Lemma 5.1.

6.1. Lemma. Let $G$ be a discrete group, $C_{r}^{*}(G)$ its reduced $C^{*}$-algebra, $Q \subset G$ a finite subset. For $g \in G$ let $\lambda(g)$ be the left regular representation and let $F$ be the orthogonal projection of $l^{2}(G)$ onto $l^{2}(Q) \subset l^{2}(G)$ and $E_{a b}(a, b \in G)$ the matrix units in $B\left(l^{2}(G)\right)$. Then there are unital completely positive maps $\varphi: C_{r}^{*}(G) \rightarrow$ $B\left(l^{2}(Q)\right)$ and $\psi: B\left(l^{2}(Q)\right) \rightarrow C_{r}^{*}(G)$ so that $\varphi(T)=F T F \mid l^{2}(Q)$ and $\psi\left(E_{a b}\right)=$ $|Q|^{-1} \lambda\left(a b^{-1}\right)$. Moreover

$$
\psi(\varphi(\lambda(g)))=|Q|^{-1}|Q \cap g Q| \lambda(g)
$$


Proof. We only sketch the proof since this is the same argument as in Lemma 5.1. By $\rho(g)$ let us denote the right regular representation. Let $K_{1} \subset K_{2} \subset \ldots, \cup_{p} K_{p}=$ $G$ be finite sets (there is no loss of generality if we assume $G$ countable). Then we define

$$
\tilde{\psi}(T)=|Q|^{-1} w-\lim _{p \rightarrow \omega} \sum_{g \in K_{p}} \rho(g) T_{\rho}\left(g^{-1}\right) \in B\left(l^{2}(G)\right)
$$

for $T \in B\left(l^{2}(Q)\right)=F B\left(l^{2}(G)\right) F$. We then find $\tilde{\psi}\left(E_{a b}\right)=|Q|^{-1} \lambda\left(a b^{-1}\right)$ and $\tilde{\psi}(\varphi(\lambda$ $(g)))=|Q|^{-1}|Q \cap g Q| \lambda(g)$ which show in particular $\tilde{\psi}$ is unital and takes values actually in $C_{r}^{*}(G) \subset B\left(l^{2}(G)\right)$.

Let $\Gamma=(Z / n Z)^{(Z)}$ and $G=\Gamma \triangleleft_{\alpha} Z$, where $\alpha$ is the shift action on $\Gamma$. Let $g \in G$ be the generator of $\mathbb{Z} \subset G$, i.e. the inner automorphism of $g$ implements the shift automorphism of $\Gamma$. There are obvious isomorphisms $C(X) \simeq C_{r}^{*}(\Gamma), C(X) \triangleleft_{\beta} \mathbb{Z} \simeq$ $C_{r}^{*}(G)$ under which $u$ corresponds to $\lambda(g)$ and our problem will be to compute $h t($ Ad $\lambda(g))$. We identify $\Gamma_{m_{1}, m_{2}}=(\mathbb{Z} / n \mathbb{Z})^{\left[m_{2}, m_{1}\right]}$ in the obvious way with a subgroup of $\Gamma \subset G$.

6.2. Proposition. We have $h t(\operatorname{Ad} u)=\log n$ for the unitary $u$ implementing the topological Benoulli shift in $C(X) \triangleleft_{\beta} \mathbb{Z}$.

Proof. It suffices to show $h t(\operatorname{Ad} u) \leqq \log n$ the opposite inequality being a consequence of 4.4 and 4.8 applied to the restriction of $\operatorname{Ad} u$ to $C(X)$. For the rest of the proof we pass to the group $G$, where the problem becomes to show $h t($ Ad $\lambda(g)) \leqq \log n$. Let $\psi_{m}, \varphi_{m}, B_{m}$ be the maps and the finite-dimensiional $C^{*}$ algebra provided by Lemma 6.1 in case

$$
Q=Q(m, k)=\left\{g^{j}|| j \mid \leqq k\right\} \Gamma_{m,-2 k} .
$$

Remark that if $0<k<m$, then $\Gamma_{m,-k,-k} Q(m, k) \subset Q(m, k)$ and hence

$$
|Q|^{-1}|Q \cap h Q| \geqq \frac{2 k+1-p}{2 k+1}
$$

if $h \in Q(m-k, p)$ with $0<p<k / 2$. It follows that

$$
\left|\psi_{m}\left(\varphi_{m}(\lambda(h))\right)-\lambda(h)\right| \leqq \frac{p}{2 k+1}
$$

if $h \in Q(m-k, p)$. Note also that $g Q(m-k, p) g^{-1} \subset Q(m+1-k, p)$. Thus if $\omega_{m}=\{\lambda(h) \mid h \in Q(m-k, p)\}$, we have $($ Ad $\lambda(g))\left(\omega_{m}\right) \subset \omega_{m+1}$. Using $\psi_{m}, \varphi_{m}, B_{m}$ we have

$$
r c p\left(\omega_{m} ; p(2 k+1)^{-1}\right) \leqq|Q(m, k)|=(2 k+1) n^{m+2 k} .
$$

Hence

$$
h t\left(\operatorname{Ad} \lambda(g), \omega_{m} ; p(2 k+1)^{-1}\right) \leqq \lim _{N \rightarrow \infty} \sup ^{-1} \log \left((2 k+1) n^{m+N+2 k}\right)=\log n .
$$

Increasing $k$ and $p$ the conclusion can now be obtained from 4.3.

6.3. Corollary. Let $\mu_{n}$ be the equal weights probability measure on $\{1, \ldots, n\}$ and let $\mu=\mu_{n}^{\otimes Z}$. Let $u$ be the implementing unitary for the Bernoulli shift action in $M=L^{\infty}(X, \mu) \triangleleft_{\beta} \mathbb{Z}$. Then

$$
H(\text { Ad } u)=\log n \text {. }
$$


Proof. Since $H(\operatorname{Ad} u) \geqq H\left(\operatorname{Ad} u \mid L^{\infty}(x, \mu)\right)=\log n$ it suffices to prove the opposite inequality

The inequality $H(\operatorname{Ad} u) \leqq \log n$ follows from 4.6 which gives $H$ Ad $u) \leqq$ $h t($ Ad $u)$ (the second Ad $u$ begin in $C(X) \triangleleft_{\beta} \mathbb{Z}$ ) and Proposition 6.2.

\section{Unitary Operators, the Hilbert Space Case}

We take a look at the approximation entropy of a unitary operator in this section.

By $\mathscr{H}$ we shall denote a complex separable Hilbert space of infinite dimension, by $\mathscr{P} f(\mathscr{H})$ the finite subsets of $\mathscr{H}$ and by $\mathscr{F}(\mathscr{H})$ the finite-dimensional subspaces of $\mathscr{H}$. If $\omega \in \mathscr{P} f(\mathscr{H})$ and $A \subset \mathscr{H}$ we shall write $\omega \subset_{\delta} A$ if for every $h \in \omega$ we can find $h^{\prime} \in A$ such that $\left\|h-h^{\prime}\right\|<\delta$. $U$ will denote a unitary operator acting on $\mathscr{H}$.

7.1. Definition. If $\omega \in \mathscr{P} f(\mathscr{H})$ and $\delta>0$, we define

$$
d(\omega ; \delta)=\inf \left\{\operatorname{dim} \chi \mid \chi \in \mathscr{F}(\mathscr{H}), \omega \subset_{\delta} \chi\right\} .
$$

7.2. Definition. If $\delta>0$ and $\omega \in \mathscr{P} f(\mathscr{H})$, we define

$$
\begin{aligned}
h(U, \omega ; \delta) & =\limsup _{n \rightarrow \infty} n^{-1} d\left(\bigcup_{0 \leqq j \leqq n-1} U^{j} \omega ; \delta\right), \\
h(U, \omega) & =\sup _{\delta>0} h(U, \omega ; \delta), \\
h(U) & =\sup \{h(U, \omega) \mid \omega \in \mathscr{P} f(\mathscr{H})\} .
\end{aligned}
$$

The reader who is by now familiar with the operator-algebra case, will easily find the proofs of the next two propositions.

7.3. Proposition. If $k \in \mathbb{Z}$ then

$$
h\left(U^{k}\right)=|k| h(U) .
$$

7.4. Proposition. Let $\omega_{j} \in \mathscr{P} f(\mathscr{H}), j \in \mathbb{N}, \omega_{1} \subset \omega_{2} \subset \ldots$ be such that $\bigcup_{j \in \mathscr{N}} \bigcup_{n \in \mathbb{Z}}$ $U^{n}\left(\omega_{j}\right)$ spans a dense subspace of $\mathscr{H}$. Then

$$
h(U)=\sup _{j \in \mathbb{N}} h\left(U, \omega_{j}\right) .
$$

7.5. Proposition. Let $\mathscr{K} \subset \mathscr{H}$ be a closed subspace such that $U \mathscr{K}=\mathscr{K}$. Then

$$
h(U \mid \mathscr{K}) \leqq h(U) .
$$

Proof. If $\omega \in \mathscr{P} f(\mathscr{K})$ and $P$ is the orthogonal projection of $\mathscr{H}$ onto $\mathscr{K}$, then if $\chi \in \mathscr{F}(\mathscr{H})$ and

$$
\omega \subset_{\delta} \chi,
$$

it follows that

$$
\omega \subset_{\delta} P \chi .
$$


Using this remark we easily infer

$$
h(U \mid \mathscr{K}, \omega ; \delta) \leqq h(U, \omega ; \delta),
$$

which then yields the desired conclusion.

7.6. Proposition. If $\mathscr{H}=\mathscr{H}_{1} \oplus \mathscr{H}_{2}$ and $U=U_{1} \oplus U_{2}$, then

$$
h(U) \leqq h\left(U_{1}\right)+h\left(U_{2}\right) .
$$

Proof. If $\omega_{j} \in \mathscr{P} f\left(\mathscr{H}_{j}\right)$ and

$$
\omega_{1} \oplus \omega_{2}=\left\{h_{1} \oplus h_{2} \mid h_{j} \in \omega_{j}, j=1,2\right\},
$$

it is easily seen that

$$
d\left(\omega_{1} \oplus \omega_{2} ; \delta_{1}+\delta_{2}\right) \leqq d\left(\omega_{1}, \delta_{1}\right)+d\left(\omega_{2}, \delta_{2}\right) .
$$

This easily yields then

$$
h\left(U, \omega_{1} \oplus \omega_{2} ; \delta_{1}+\delta_{2}\right) \leqq h\left(U_{1}, \omega_{1} ; \delta_{1}\right)+h\left(U_{2}, \omega_{2} ; \delta_{2}\right),
$$

and then the desired conclusion.

With these preparations we begin proving the formula for $h(U)$ via a sequence of lemmas. We will extensively use facts from [12].

7.7. Lemma. If the spectral measure of $U$ is singular with respect to Lebesgue measure then

$$
h(U)=0 .
$$

Proof. Lemma 5.1 of [12] says precisely that given $\varepsilon>0$ and $\omega \in \mathscr{P} f(\mathscr{H})$ there is $k_{0} \in \mathbb{N}$ so that if $k \geqq k_{0}$, then

$$
d\left(\omega \cup \cdots \cup U^{k-1} \omega ; \varepsilon\right) \leqq k \varepsilon .
$$

This clearly gives the desired conclusion.

7.8. Lemma. If $\omega=\left\{e_{1}, \ldots, e_{n}\right\}$ is an orthonormal system of vectors and $\varepsilon>0$, then

$$
d(\omega ; \varepsilon) \geqq n\left(1-\varepsilon^{2}\right) .
$$

Proof. Assume $\omega \subset_{\varepsilon} \mathscr{H}_{1}, \mathscr{H}_{1} \in \mathscr{F}(\mathscr{H})$. Replacing $\mathscr{H}_{1}$ by $P \mathscr{H}_{1}$, where $P$ is the orthogonal projection onto $\mathbb{C} e_{1}+\cdots+\mathbb{C} e_{n}$ we clearly have $\omega \subset_{\varepsilon} P \mathscr{H}_{1}$ and $\operatorname{dim}$ $P \mathscr{H}_{1} \leqq \operatorname{dim} \mathscr{H}_{1}$. Thus, we may assume $\mathscr{H}_{1} \subset \mathbb{C} e_{1}+\cdots+\mathbb{C} e_{n}$. Denoting by $Q$ the orthogonal projection onto $\mathscr{H}_{1}$ we have

$$
\begin{aligned}
n-\operatorname{Tr} Q & =\operatorname{Tr}(P-Q) \\
& =\sum_{1 \leqq j \leqq n}\left\langle(P-Q) e_{j}, e_{j}\right\rangle \\
& =\sum_{1 \leqq j \leqq n}\left\|(P-Q) e_{j}\right\|^{2} \leqq n \varepsilon^{2} .
\end{aligned}
$$

Hence $\operatorname{dim} \mathscr{H}_{1}=\operatorname{Tr} Q \geqq n\left(1-\varepsilon^{2}\right)$. 
7.9. Lemma. If $U$ is a bilateral shift of multiplicity $n$ then

$$
h(U)=n \text {. }
$$

Proof. Let $\omega$ be an orthonormal basis for a wandering subspace of $U$. By 7.8 we have

$$
h(U, \omega ; \delta) \geqq n\left(1-\delta^{2}\right),
$$

and on the other hand it is obvious that $h(U, \omega ; \delta) \leqq n$. This clearly yields the desired conclusion.

7.10. Lemma. Let $\mathscr{K}_{1} \subset \mathscr{K}_{2} \subset \ldots$ be closed subspaces of $\mathscr{H}$ such that $U \mathscr{K}_{j}=$ $\mathscr{K}$, and $\bigcup_{j \in \mathbb{N}} \mathscr{K}_{j}$ is dense in $\mathscr{H}$. Then

$$
h\left(U \mid \mathscr{K}_{j}\right) \uparrow h(U) .
$$

Proof. Let $\omega_{j} \in \mathscr{P} f\left(\mathscr{K}_{j}\right), \omega_{1} \subset \omega_{2} \subset \ldots$ be such that $\bigcup_{j \in \mathbb{N}} \omega_{j}$ spans a dense subspace in $\mathscr{H}$. By 7.4 and 7.5

$$
\sup _{j \in \mathbb{N}} h\left(U, \omega_{j}\right)=h(U),
$$

$h\left(U \mid \mathscr{K}_{j}\right)$ is increasing and

$$
h\left(U, \omega_{j}\right) \leqq h\left(U \mid \mathscr{K}_{j}\right) \leqq h(U),
$$

which yields the desired conclusion.

7.11. Proposition. Let $\mathbb{T}=\{z \in \mathbb{C}|| z \mid=1\}$ and $m: \mathbb{T} \rightarrow \mathbb{N} \cup\{0\}$ be the multiplicity function of the Lebesgue absolutely continuous part of $U$. Then

$$
h(U)=\int_{\mathbb{T}} m(z) d \lambda(z),
$$

where $d \lambda$ is normalized Haar measure.

Proof. We will use the machinery from the case of Bogoliubov automorphisms in [12].

Remark that if $U=U_{a} \oplus U_{s}$ is the decomposition of $U$ into absolutely continuous and singular parts, then by $7.5,7.6$ and 7.7 and we have

$$
h\left(U_{a}\right) \leqq h(U) \leqq h\left(U_{a}\right)+h\left(U_{s}\right)=h\left(U_{a}\right),
$$

so that $h(U)=h\left(U_{a}\right)$. So it will suffice to prove the proposition in case $U=U_{a}$. Clearly $h(U)$ is then a function of $m$ and defines a map $\mu: \mathscr{C} \rightarrow \mathbb{R}^{+}$, where $\mathscr{C}$ is the additive semigroup of functions $f: \mathbb{T} \rightarrow\{0\} \cup \mathbb{N}$ which are Lebesgue measurable. Let $\mathbb{1}$ be the constant function equal to 1 on $\mathbb{T}$ and $T_{n}: \mathscr{C} \rightarrow \mathscr{C}$ the map

$$
\left(T_{n} f\right)(\rho)=\sum_{x^{n}=\rho} f(z) .
$$

We shall use Theorem 2.1 in [12] to prove our assertion. For this we check the following conditions: 
(i) $\mu(n \mathbb{1})=n$. This follows from Lemma 7.9.

(ii) $f \leqq g \Rightarrow \mu(f) \leqq \mu(g)$. This follows from Proposition 7.5.

(iii) $f_{j} \uparrow f \Rightarrow \mu\left(f_{j}\right) \uparrow \mu(f)$. This follows from Lemma 7.10.

(iv) $\mu\left(T_{n} f\right)=n \mu(f)$. This follows from Proposition 7.3.

(v) $\mu(f)=\mu(g)$ if $f$ and $g$ are equal a.e. w.r.t. Lebesgue measure. This is obvious.

Having checked these conditions, Theorem 2.1 of [12] says precisely that $\mu(f)=$ $\int_{\mathbb{T}} f(z) d \lambda(z)$.

7.12. Remark. The preceding proposition shows that up to a proportionality constant (depending on the choice of basis of logarithms) $h(U)$ coincides with the entropy $h_{r}\left(\alpha_{U}\right)$ of the induced Bogoliubov automorphism $\alpha_{U}$ with respect to the unique trace state on the $C A R$-algebra (see [12]).

7.13. Proposition. If $\delta>0$ and $\omega \in \mathscr{P} f(\mathscr{H})$, then

$$
h(U, \omega ; \delta)=\lim _{n \rightarrow \infty} n^{-1} d\left(\bigcup_{0 \leqq j \leqq n-1} U^{j} \omega ; \delta\right) .
$$

Proof. We have

$$
\begin{aligned}
d\left(\bigcup_{0 \leqq j \leqq n+m-1} U^{j} \omega ; \delta\right) & \leqq d\left(\bigcup_{0 \leqq j \leqq n-1} U^{j} \omega ; \delta\right)+d\left(\bigcup_{n \leqq j \leqq n+m-1} U^{j} \omega ; \delta\right) \\
& =d\left(\bigcup_{0 \leqq j \leqq n-1} U^{j} \omega ; \delta\right)+d\left(\bigcup_{0 \leqq j \leqq m-1} U^{j} \omega ; \delta\right)
\end{aligned}
$$

which implies that $n^{-1} d\left(\bigcup_{0 \leqq j \leqq n-1} U^{j} \omega ; \delta\right)$ is convergent.

\section{Representations of Amenable Discrete Groups}

Here we generalize the context of the preceding section from representations of $\mathbb{Z}$ to representations of certain amenable discrete groups. In the case of representations quasiequivalent to the regular representation of an i.c.c. group, the entropy equals the von Neumann dimension (Proposition 8.8).

By $G$ we denote an infinite discrete group with a system of generators $S$ and $\|g\|$ will denote the minimal length of a word in the generators $S$ representing $g$. Let $K_{n}=\{g \in G \mid\|g\| \leqq n\}$, we shall assume $G$ satisfies $\lim _{n \rightarrow \infty}\left|K_{n}\right|\left|K_{n+1}\right|^{-1}=1$ (clearly this implies $G$ is amenable).

By $\mathscr{H}$ we shall denote a separable complex Hilbert space of infinite dimension, $U(\mathscr{H})$ the unitary operators on $\mathscr{H}$ and $\pi: G \rightarrow U(\mathscr{H})$ a representation. 
8.1. Definition. If $\delta>0$ and $\omega \in \mathscr{P} f(\mathscr{H})$ we define

$$
\begin{aligned}
h(\pi, \omega ; \delta) & =\limsup _{n \rightarrow \infty}\left|K_{n}\right|^{-1} d\left(\pi\left(K_{n}\right) \omega ; \delta\right), \\
\operatorname{lh}(\pi, \omega ; \delta) & =\liminf _{n \rightarrow \infty}\left|K_{n}\right|^{-1} d\left(\pi\left(K_{n}\right) \omega ; \delta\right), \\
h(\pi, \omega) & =\sup _{\delta>0} h(\pi, \omega ; \delta), \\
l h(\pi, \omega) & =\sup _{\delta>0} \ln (\pi, \omega ; \delta), \\
h(\pi) & =\sup \{h(\pi, \omega) \mid \omega \in \mathscr{P} f(\mathscr{H})\}, \\
\operatorname{lh}(\pi) & =\sup \{\operatorname{lh}(\pi, \omega) \mid \omega \in \mathscr{P} f(\mathscr{H})\} .
\end{aligned}
$$

$h(\pi)$ will be called the entropy of $\pi$ and $\operatorname{lh}(\pi)$ the lower entropy of $\pi$.

The proofs of the next three propositions are quite standard and will be omitted.

8.2. Proposition. Let $\omega_{j} \in \mathscr{P} f(\mathscr{H}), j \in \mathbb{N}, \omega_{1} \subset \omega_{2} \subset \ldots$ be such that $\bigcup_{j \in \mathbb{N}}$ $\bigcup_{g \in G} \pi(g) \omega_{j}$ spans a dense subspace of $\mathscr{H}$. Then

$$
\begin{gathered}
h(\pi)=\sup _{j \in \mathbb{N}} h\left(\pi, \omega_{j}\right), \\
\operatorname{lh}(\pi)=\sup _{j \in \mathbb{N}} \ln \left(\pi, \omega_{j}\right) .
\end{gathered}
$$

(Note that here our assumption on the growth of $G$ is essential.)

8.3. Proposition. If $\pi=\pi_{1} \oplus \pi_{2}, \mathscr{H}=\mathscr{H}_{1} \oplus \mathscr{H}_{2}, \omega_{j} \in \mathscr{P} f\left(\mathscr{H}_{j}\right)$, then

$$
\begin{aligned}
h\left(\pi_{1} \oplus \pi_{2},\left(\omega_{1} \oplus 0\right) \cup\left(0 \oplus \omega_{2}\right) ; \delta\right) & \leqq h\left(\pi_{1}, \omega_{1} ; \delta\right)+h\left(\pi_{2}, \omega_{2} ; \delta\right), \\
h\left(\pi_{1} \oplus \pi_{2},\left(\omega_{1} \oplus 0\right) \cup\left(0 \oplus \omega_{2}\right)\right) & \leqq h\left(\pi_{1}, \omega_{1}\right)+h\left(\pi_{2}, \omega_{2}\right), \\
h\left(\pi_{1} \oplus \pi_{2}\right) & \leqq h\left(\pi_{1}\right)+h\left(\pi_{2}\right) .
\end{aligned}
$$

8.4. Proposition. Let $\mathscr{K} \subset \mathscr{H}, \mathscr{K}_{j} \subset \mathscr{H}$ be $\pi(G)$ invariant closed subspaces and assume $\mathscr{K}_{1} \subset \mathscr{K}_{2} \subset \ldots, \bigcup_{j \in \mathbb{N}} \mathscr{K}_{j}=\mathscr{H}$. Then

$$
\begin{aligned}
h(\pi \mid \mathscr{K}) & \leqq h(\pi), \\
\operatorname{lh}(\pi \mid \mathscr{K}) & \leqq \operatorname{lh}(\pi), \\
h(\pi) & =\sup _{j \in \mathbb{N}} h\left(\pi \mid \mathscr{K}_{j}\right), \\
\operatorname{lh}(\pi) & =\sup _{j \in \mathbb{N}} \ln \left(\pi \mid \mathscr{K}_{j}\right) .
\end{aligned}
$$

8.5. Lemma. Let $\mathscr{H}=\mathscr{H}_{1} \oplus \mathscr{H}_{2}, \omega_{j} \in \mathscr{P} f\left(\mathscr{H}_{j}\right)$ be such that $\xi \in \omega_{j} \Rightarrow 0<C_{1}$ $\leqq\|\xi\| \leqq C_{2}$. Let further $0<\delta<C_{1}$. Then

$$
d\left(\left(\omega_{1} \oplus 0\right) \cup\left(0 \oplus \omega_{2}\right) ; \delta C_{2}^{-1}\right) \geqq d\left(\omega_{1} ; C_{1}^{-1} \sqrt{4 \delta}\right)+d\left(\omega_{2} ; C_{1}^{-1} \sqrt{4 \delta}\right) .
$$

Proof. Replacing $\omega_{j}$ by

$$
\tilde{\omega}_{j}=\left\{\|\xi\|^{-1} \xi \mid \xi \in \omega_{j}\right\}
$$


it is easily seen that the lemma under the more restrictive conditions $C_{1}=C_{2}=1$ implies the lemma in the general case.

Thus we shall assume $\xi \in \omega_{j} \Rightarrow\|\xi\|=1$ and $0<\delta<1$.

Let $\chi \in \mathscr{F}\left(\mathscr{H}_{1} \oplus \mathscr{H}_{2}\right)$ be such that

$$
\left(\omega_{1} \oplus 0\right) \cup\left(0 \oplus \omega_{2}\right) \subset_{\delta} \chi
$$

Let $Q$ be the projection onto $\chi$, and $P_{1}, P_{2}$ the projections onto $\mathscr{H}_{1} ; \mathscr{H}_{2}$. Let further

$$
\omega_{1}^{\prime}=Q\left(\omega_{1} \oplus 0\right), \omega_{2}^{\prime}=Q\left(0 \oplus \omega_{2}\right),
$$

and $A=Q P_{1} Q \mid \chi$. If $\xi \in \omega_{1} \oplus 0$, then

$$
\|(I-Q) \xi\|<\delta
$$

Denoting $Q \xi=\eta$, we have

$$
\|\eta\|^{2} \geqq 1-\delta^{2}
$$

and

$$
\begin{aligned}
\langle A \eta, \eta\rangle & =\left\|P_{1} Q \xi\right\|^{2} \\
& \geqq\left(\|\xi\|-\left\|P_{1}(I-Q) \xi\right\|\right)^{2} \\
& \geqq(1-\delta)^{2} .
\end{aligned}
$$

Hence denoting by $E(A ; \cdot)$ the spectral measure of $A$, we have

$$
(1-\delta)^{2} \leqq\left\langle\left(I-\frac{1}{2} E\left(A ;\left[0, \frac{1}{2}\right]\right) \eta, \eta\right\rangle \leqq 1-\frac{1}{2}\left\|E\left(A ;\left[0, \frac{1}{2}\right]\right) \eta\right\|^{2}\right.
$$

so that

$$
\left\|E\left(A ;\left[0, \frac{1}{2}\right]\right) \eta\right\|^{2} \leqq 2\left(1-(1-\delta)^{2}\right)=4 \delta-2 \delta^{2}
$$

This means

$$
\left\|\eta-E\left(A ;\left(\frac{1}{2}, 1\right]\right) \eta\right\|^{2} \leqq 4 \delta-2 \delta^{2}
$$

so that

$$
\omega_{1}^{\prime} \subset \sqrt{4 \delta-2 \delta^{2}} E\left(A ;\left(\frac{1}{2}, 1\right]\right) \chi .
$$

Similarly, since $Q P_{2} Q \mid \chi=I-A$ we find

$$
\omega_{2}^{\prime} \subset \sqrt{4 \delta-2 \delta^{2}} E\left(A ;\left[0, \frac{1}{2}, 1\right)\right) \chi .
$$

This in turn gives

$$
\begin{aligned}
& \omega_{1} \oplus 0 \subset_{\sqrt{4 \delta}} E\left(A ;\left(\frac{1}{2}, 1\right]\right) \chi, \\
& 0 \oplus \omega_{2} \subset_{\sqrt{4 \delta}} E\left(A ;\left[0, \frac{1}{2}\right)\right) \chi,
\end{aligned}
$$


and hence

$$
\begin{aligned}
& \operatorname{dim} E\left(A ;\left(\frac{1}{2}, 1\right]\right) \chi \geqq d\left(\omega_{1} ; \sqrt{4 \delta}\right), \\
& \operatorname{dim} E\left(A ;\left[0, \frac{1}{2}\right)\right) \chi \geqq d\left(\omega_{2} ; \sqrt{4 \delta}\right),
\end{aligned}
$$

so that

$$
\operatorname{dim} \chi \geqq d\left(\omega_{1} ; \sqrt{4 \delta}\right)+d\left(\omega_{2} ; \sqrt{4 \delta}\right) .
$$

8.6. Proposition. Let $\mathscr{H}=\mathscr{H}_{1} \oplus \mathscr{H}_{2}, \omega_{j} \in \mathscr{P} f\left(\mathscr{H}_{j}\right)$ be such that $\xi \in \omega_{j} \Rightarrow 0<$ $C_{1} \leqq\|\xi\| \leqq C_{2}$. Let further $0<\delta<C_{1}$. If $\pi_{j}$ are unitary representations of $G$ on $\mathscr{H}_{j}$, then

$$
\begin{aligned}
& \operatorname{lh}\left(\pi_{1} \oplus \pi_{2},\left(\omega_{1} \oplus 0\right) \cup\left(0 \oplus \omega_{2}\right) ; \delta C_{2}^{-1}\right) \geqq \operatorname{lh}\left(\pi_{1}, \omega_{1} ; C_{1}^{-1} \sqrt{4 \delta}\right) \\
&+\operatorname{lh}\left(\pi_{2}, \omega_{2} ; C_{2}^{-1} \sqrt{4 \delta}\right), \\
& \operatorname{lh}\left(\pi_{1} \oplus \pi_{2},\left(\omega_{1} \oplus 0\right) \cup\left(0 \oplus \omega_{2}\right)\right) \geqq \operatorname{lh}\left(\pi_{1}, \omega_{1}\right)+\ln \left(\pi_{2}, \omega_{2}\right), \\
& \operatorname{lh}\left(\pi_{1} \oplus \pi_{2}\right) \geqq \ln \left(\pi_{1}\right)+\operatorname{lh}\left(\pi_{2}\right) .
\end{aligned}
$$

Proof. The first inequality follows immediately from 8.5 . For the second it suffices to notice that $\operatorname{lh}(\pi, \omega ; \delta)$ is a decreasing function of $\delta$. For the last inequality one uses 8.2 and the fact that $\ln (\pi, \omega)$ is an increasing function of $\omega$.

8.7. Proposition. Let $\pi$ be a unitary representation of $G$ on $\mathscr{H}$ and $\omega \in \mathscr{P} f(\mathscr{H})$, so that $\|\xi\|=1$ for all $\xi \in \omega$. If $\delta>0$ and $\delta_{n}=2^{n} \delta^{2^{-n}}$, then denoting $\pi \oplus \cdots \oplus$ $\pi=\pi \otimes 1_{n}(n$ copies of $\pi)$ and by $\omega_{n}$ the union of the $0 \oplus \ldots 0 \oplus \omega \oplus 0 \ldots \oplus 0$, where $\omega$ appears in the $j^{\text {th }}$ position $(1 \leqq j \leqq n)$. Then

$$
\begin{aligned}
n l h\left(\pi, \omega ; \delta_{n}\right) & \leqq \operatorname{lh}\left(\pi \otimes 1_{n}, \omega_{n} ; \delta\right) \\
& \leqq n \operatorname{lh}(\pi, \omega ; \delta), \\
n h\left(\pi, \omega ; \delta_{n}\right) & \leqq h\left(\pi \otimes 1_{n}, \omega_{n} ; \delta\right) \\
& \leqq n h(\pi, \omega ; \delta), \\
n l h(\pi, \omega) & =\operatorname{lh}\left(\pi \otimes 1_{n}, \omega_{n}\right), \\
n h(\pi, \omega) & =h\left(\pi \otimes 1_{n}, \omega_{n}\right), \\
n l h(\pi) & =\ln \left(\pi \otimes 1_{n}\right), \\
n h(\pi) & =h\left(\pi \otimes 1_{n}\right) .
\end{aligned}
$$

Proof. All this follows easily from $n d\left(\omega ; \delta_{n}\right) \leqq d\left(\omega_{n} ; \delta\right) \leqq n d(\omega, \delta)$ which is a consequence of 8.5 .

8.8. Proposition. Let $\lambda_{G}$ be the left regular representation of $G$ on $l^{2}(G)$. Then

$$
\ln \left(\lambda_{G}\right)=h\left(\lambda_{G}\right)=1 \text {. }
$$


If $G$ is an i.c.c. group and $\pi$ is quasiequivalent to $\lambda_{g}$ let $m$ be the von Neumann dimension of $\pi$. Then

$$
\operatorname{lh}(\pi)=h(\pi)=m \text {. }
$$

Proof. In $l^{2}(G)$ let $\xi$ be the vector so that $\xi(g)=\delta_{g, \varepsilon}$. Then Lemma 7.8 gives

$$
\left(1-\delta^{2}\right)\left|K_{n}\right| \leqq d\left(\pi\left(K_{n}\right) \xi ; \delta\right) \leqq\left|K_{n}\right|,
$$

which easily gives $l h\left(\lambda_{g}\right)=h\left(\lambda_{g}\right)=1$ using 8.2.

If $m$ is an integer the second assertion follows from 8.7. Since a rational number has an integral multiple applying again 8.7 we infer the second assertion if $m$ is rational.

The general case follows now since $l h(\pi)$ and $h(\pi)$ are increasing functions of $m$ by 8.4 .

\section{Further Remarks}

This section is a collection of remarks about problems concerning approximation: restrictions to invariant subalgebras, tensor products and last but not least the dimension versus rank question.

\subsection{Restrictions.}

The problem is, whether

$$
h a(\alpha \mid N) \leqq h a(\alpha),
$$

where $N \subset M$ is a von Neumann subalgebra of $M$, so that $\alpha(N)=N$. There is an obvious analogue of this question for hat in the $A F$-algebra context. Progress on this question may involve developing further the techniques of Christensen [2].

\subsection{Tensor Products.}

Propositions $1.9,2.7,3.10$ and 4.9 naturally lead to the question whether for any of these entropies the entropy of $\alpha \otimes \beta$ actually equals the sum of those of $\alpha$ and $\beta$.

\subsection{Dimension versus Rank.}

In the definitions of $h a$ and hat one may replace the $\delta$-rank by the $\delta$-dimension, thereby obtaining two invariants $h a d(\alpha)$ and $h a d t(\alpha)$. In more details, we define

$$
d(\omega ; \delta)=\inf \left\{\operatorname{dim} A \mid A \in \mathscr{F}(M), \omega \subset_{\delta} A\right\},
$$

where the $\delta$-inclusion is w.r.t. | $\mid 2$ in the $W^{*}$-case and w.r.t \|\| in the $A F$ case. One then defines $\operatorname{had}(\alpha, \omega ; \delta), \operatorname{had}(\alpha, \omega), \operatorname{had}(\alpha)$ like in Definition 1.2 and $\operatorname{hadt}(\alpha, \omega ; \delta), \operatorname{hadt}(\alpha, \omega), \operatorname{hadt}(\alpha)$ like in Definition 2.1 with $r(\omega ; \delta)$ replaced by $d(\omega ; \delta)$. 
Similarly one can modify Definition 3.1,

$d c p_{\sigma}(\omega ; \delta)=\inf \left\{\operatorname{dim} B \mid(\varphi, \psi, B) \in C P A(M, \sigma),\|(\psi \circ \varphi)(a)-a\|_{\sigma}<\delta\right.$ for $\left.a \in \omega\right\}$, and the definition in Sect. 4

$$
d c p(\omega ; \delta)=\inf \{\operatorname{dim} B \mid(\varphi, \psi, B) \in C P A(M),\|(\psi \circ \varphi)(a)-a\|<\delta \text { for } a \in \omega\} .
$$

This can then be followed up with definitions of $\operatorname{hcpad}_{\sigma}(\alpha, \omega ; \delta), \operatorname{hcpad}_{\sigma}(\alpha, \omega)$, $\operatorname{hcpad}_{\sigma}(\alpha)$ and respectively $h t d(\alpha, \omega ; \delta), h t d(\alpha, \omega), h t d(\alpha)$ by replacing $r c p$ with $d c p$.

Since $\operatorname{rank} A \leqq \operatorname{dim} A \leqq(\operatorname{rank} A)^{2}$ and $\operatorname{rank} A$ equals $\operatorname{dim} A$ if $A$ is commutative, we infer: $h a(\alpha) \leqq h a d(\alpha) \leqq 2 h a(\alpha)$, hat $(\alpha) \leqq h a t d(\alpha) \leqq 2 h a t d(\alpha), h c p a_{\sigma}(\alpha)$ $\leqq \operatorname{hpad}_{\sigma}(\alpha) \leqq 2 \operatorname{hcpad}_{\sigma}(\alpha), h t(\alpha) \leqq h t d(\alpha) \leqq 2 h t(\alpha)$. If $A$ is commutative the lower bounds for $\operatorname{had}(\alpha), \operatorname{hadt}(\alpha), \operatorname{hrpad}_{\sigma}(\alpha), \operatorname{htd}(\alpha)$ are attained. It is a natural question whether for Bernoulli shifts $2 h a(\alpha)=\operatorname{had}(\alpha), 2 \operatorname{hat}(\alpha)=\operatorname{hatd}(\alpha), 2 h c p a_{\sigma}$ $(\alpha)=h \operatorname{cpad}_{\sigma}(\alpha), 2 h t(\alpha)=h t d(\alpha)$. It is also natural to ask whether there are ergodic automorphisms of the hyperfinite $\mathrm{II}_{1}$-factor for which $h a(\alpha)=\lambda h a d(\alpha)$ or $h c p a_{\tau}$ $(\alpha)=\lambda \operatorname{hopad}_{\tau}(\alpha)$ with $1<\lambda<2$.

\subsection{Miscellaneous.}

There are several natural extensions to consider. Most of the facts about entropies of automorphisms of operator algebras work for endomorphisms. In another direction the definition of the entropy of a unitary operator easily adapts to a definition of an entropy for isometric automorphisms of Banach spaces.

\section{Appendix. A Question of Störmer on Implemented Ergodic Transformations}

Using the completely positive approximation entropy of Sect. 3 we answer here a question of Störmer (Problem 4.2 in [11]). The result solving the problem is the Corollary at the end of this Appendix.

Let $(X, \mu)$ be a probability measure space and let $\alpha$ be the automorphism of $L^{\infty}(X, \mu)$ induced by an ergodic measure-preserving transformation $T$ of $(X, \mu)$. On $\mathscr{M}=L^{\infty}(X, \mu) \triangleleft_{\alpha} \mathbb{Z}$ let $\tau$ be the trace-state corresponding to $\mu$. Let $\mathscr{A} \subset L^{\infty}(X, \mu)$ be a unital finite-dimensional subalgebra (i.e. the functions measurable w.r.t. a finite measurable partition). We denote by $\mathscr{H}_{n}$ the subspace of $L^{2}(\mathscr{M}, \tau)$,

$$
\mathscr{H}_{n}=\sum_{|k| \leqq n} u^{k} \mathscr{A}
$$

and by $Q_{n}$ the orthogonal projection of $L^{2}(\mathscr{M}, \tau)$ onto $\mathscr{H}_{n}$. Let $P_{k}$ be the orthogonal projection of $\mathscr{H}_{n}$ onto $u^{k} \mathscr{A}(|k| \leqq n)$ and $e_{k l}$ the partial isometry from $u^{\prime} \mathscr{A}$ to $u^{k} \mathscr{A}$ determined by left multiplication by $u^{k-l}$. We also denote by $L_{x}$ and $R_{x}$ the left and right multiplication operators by $x \in \mathscr{M}$ on $L^{2}(\mathscr{M}, \tau)$.

\section{Lemma 1.}

$$
Q_{n} L_{u^{m} f} Q_{n}=\sum_{\substack{|k| \leqq n \\|k+m| \leqq n}} e_{k+m, k} R_{E\left(x^{-1} f\right)} P_{k}
$$


where $f \in L^{\infty}(x, \mu)$ and $E$ is the conditional expectation of $L^{\infty}(X, \mu)$ onto $\mathscr{A}$. In particular

$$
Q_{n} L_{\mathscr{M}} Q_{n} \subset \sum_{\substack{|k| \leqq n \\|l| \leqq n}} e_{k l} R_{\mathscr{A}} \simeq B\left(l^{2}([-n, n]) \otimes \mathscr{A} .\right.
$$

Proof. This is a quite standard computation, which we shall only sketch.

If $g \in \mathscr{A}$, then

$$
L_{u^{m}} u^{k} g=u^{m+k} \alpha^{-k}(f) g
$$

and hence

$$
\begin{aligned}
P_{r} L_{u^{m} f} u^{k} g & =L_{u^{r}} P_{0} L_{u^{-r}} u^{m+k} \alpha^{-k}(f) g \\
& =L_{u^{r}} P_{0} u^{m+k-r} \alpha^{-k}(f) g \\
& = \begin{cases}0 & \text { if } m+k \neq r \\
u^{m+k} E\left(\alpha^{-k}(f)\right) g & \text { if } r=m+k,\end{cases}
\end{aligned}
$$

so that

$$
P_{r} L_{u^{m} f} P_{k}= \begin{cases}0 & \text { if } m+k \neq r \\ e_{r k} R_{E\left(\alpha^{-k}(f)\right)} & \text { if } m+k=r\end{cases}
$$

Since $Q_{n}=\sum_{|k| \leqq n} P_{k}$ we easily get the desired result.

Lemma 2. There is a unital completely positive map

$$
\psi_{n}: B\left(l^{2}([-n, n])\right) \otimes \mathscr{A} \rightarrow \mathscr{M},
$$

so that

$$
\psi_{n}\left(e_{k l} \otimes f\right)=(2 n+1)^{-1} u^{k-l} \alpha^{l}(f)
$$

Proof. Since $\mathscr{A} \subset L^{\infty}(X, \mu)$, it will suffice to prove there are completely positive maps

$$
\tilde{\psi}_{n}: B\left(l^{2}([-n, n])\right) \otimes L^{\infty}(X, \mu) \rightarrow \mathscr{M},
$$

so that

$$
\tilde{\psi}_{n}\left(e_{k l} \otimes f\right)=(2 n+1)^{-1} u^{k-l} \alpha^{l}(f) .
$$

Replacing in the construction of Lemma $1, \mathscr{A}$ by $L^{\infty}(X, \mu)$ we get a projection $\tilde{Q}_{n}$ of $L^{2}(\mathscr{M}, \tau)$ onto $\sum_{|k| \leqq n} u^{k} L^{2}(X, \mu)$ and partial isometries $\tilde{e}_{k l}$ from $u^{l} L^{2}(X, \mu)$ to $u^{k} L^{2}$ $(X, \mu)$ via left multiplication by $u^{k-l}$. Lemma 1 becomes now

$$
\tilde{Q}_{n} L_{u^{m} f} \tilde{Q}_{n}=\sum_{\substack{|k| \leqq n \\|k+m| \leqq n}} \tilde{e}_{k+m, k} R_{\alpha-k} \tilde{P}_{k}
$$

where $\tilde{P}_{k}$ is the projection onto $u^{k} L^{2}(X, \mu)$ and

$$
\tilde{Q}_{n} L, \mu \tilde{Q}_{n} \subset \sum_{\substack{|k| \leqq n \\|l| \leqq n}} \tilde{e}_{k l} R_{L^{\infty}(X, \mu)} \simeq B\left(l^{2}([-n, n])\right) \otimes L^{\infty}(X, \mu) .
$$

Thus $B\left(l^{2}([-n, n])\right) \otimes L^{\infty}(X, \mu)$ identifies with a subalgebra of $B\left(L^{2}(\mathscr{M}, \tau)\right)$ and we define

$$
\tilde{\psi}_{n}(K)=(2 n+1)^{-1} \sum_{k \in \mathbb{Z}} R_{u^{k}} K R_{u^{-k}}
$$


Note that $R_{u} p \tilde{e}_{k l} R_{u^{-p}}=\tilde{e}_{k+p, l+p}$, since

$$
\begin{aligned}
R_{u} p \tilde{e}_{k l} R_{u-p} u^{p+l} g & =R_{u} p \tilde{e}_{k l} u^{l} \alpha^{p}(g) \\
& =R_{u} p u^{k} \alpha^{p}(g)=u^{k+p} g=\tilde{e}_{k+p, l+p} U^{p+l} g
\end{aligned}
$$

(however, the analogous formula for $e_{k, l}$ doesn't hold!). Hence

$$
\begin{aligned}
\tilde{\psi}_{n}\left(e_{k l} \otimes f\right) & =(2 n+1)^{-1} \sum_{p \in Z} R_{u} p\left(\tilde{e}_{k l} R_{f}\right) R_{u^{-p}} \\
& =(2 n+1)^{-1} \sum_{p \in Z} R_{u} \tilde{e}_{k l} L_{\alpha^{l}(f)} R_{u^{-p}} \\
& =(2 n+1)^{-1} \sum_{p \in Z} \tilde{e}_{k+p, l+p} L_{\alpha^{l}(f)} \\
& =(2 n+1)^{-1} L_{\alpha^{k-l} \alpha^{l}(f)}
\end{aligned}
$$

Also, clearly $\tilde{\psi}_{n}$ is completely positive and

$$
\tilde{\psi}_{n}(1)=\tilde{\psi}_{n}\left(\sum_{-n \leqq k \leqq n} \tilde{e}_{n n}\right)=(2 n+1)(2 n+1)^{-1} L_{u^{0}}=1
$$

Combining Lemma 1 and 2 we immediately get

Lemma 3. Let

$$
\varphi_{n}: \mathscr{M} \rightarrow B\left(l^{2}([-n, n])\right) \otimes \mathscr{A}
$$

be the unital completely positive map defined by

$$
\varphi_{n}(x)=Q_{n} L_{x} Q_{n} \in \sum_{\substack{|k| \leqq n \\|l| \leqq n}} e_{k l} R_{\mathscr{A}} \simeq B\left(l^{2}([-n, n])\right) \otimes \mathscr{A}
$$

Let further $\psi_{n}: B\left(l^{2}([-n, n])\right) \otimes \mathscr{A} \rightarrow \mathscr{M}$ be the unital completely positive map in Lemma 2. We have

$$
\tau\left(\left(\psi_{n} \circ \varphi_{n}\right)(x)\right)=\tau(x)
$$

and

$$
\left(\psi_{n} \circ \varphi_{n}\right)\left(u^{m} g\right)= \begin{cases}0 & \text { if }|m|>|2 n| \\ (2 n+1)^{-1} \sum_{\substack{|k| \leqq n \\|k+m| \leqq n}} u^{m} \alpha^{k}\left(E\left(\alpha^{-k}(g)\right)\right) & \text { if }|m| \leqq|2 n|\end{cases}
$$

Proposition. We have

$$
h c p a_{\tau}(\operatorname{Ad} u) \leqq h(T)
$$

Proof. Let $\Omega=\left(\Omega_{1}, \ldots, \Omega_{m}\right)$ be a measurable partition of $X$ and $\chi_{\Omega_{J}}$ the corresponding indicator functions. It will be sufficient in view of Proposition 3.4 to show that

$$
h c p a_{\tau}\left(\operatorname{Ad} u, w_{N}\right) \leqq h(T)
$$


for $w_{N}=\left\{u^{k} \chi_{\Omega_{j}}|| k \mid \leqq N, 1 \leqq j \leqq m\right\}$. Like in the proof of Proposition 1.7 we use McMillan's theorem to construct for large subalgebras $A_{s}$ such that

$$
\alpha^{j}\left(w_{0}\right) \subset_{e^{1 / 2}} A_{s} \text { for } 0 \leqq j<s
$$

and $\operatorname{dim} A_{s} \leqq 1+\exp (s(h(T)+\varepsilon))$. To evaluate

$$
r c p_{\tau}\left(w_{N} \cup \cdots \cup(\operatorname{Ad} u)^{s-1}\left(w_{N}\right) ; \delta\right)
$$

we use Lemma 3 . In the construction of Lemma 3 choose $n=C N$ and $\mathscr{A}=$ $\alpha^{-n}\left(A_{s+3 n}\right)$. Then an element in $w_{n} \cup \cdots \cup(\operatorname{Ad} u)^{s-1}\left(w_{N}\right)$ is of the form $u^{m} \alpha^{t}\left(\chi_{\Omega_{j}}\right)$ with $0 \leqq t<s$ and $|m| \leqq N$. Then the set $\{k|| k|\leqq n| k+m \mid, \leqq n\}$ has $\geqq(2 C-$ 2) $N$ elements. With the notations of Lemma 3

$$
\begin{gathered}
\mid \alpha^{k}\left(E \left(\alpha^{-k}\left(\alpha^{t}\left(\chi_{\Omega_{j}}\right)\right)-\alpha^{t}\left(\chi_{\Omega_{j}}\right) \mid<\varepsilon^{1 / 2},\right.\right. \\
(0 \leqq t<s,|k| \leqq n,|k+m| \leqq n),
\end{gathered}
$$

since

$$
\alpha^{-k+1}\left(\chi_{\Omega_{j}}\right) \subset_{e^{1 / 2}} \alpha^{-1}\left(A_{s+3 n}\right) .
$$

(Indeed $0 \leqq t+(n-k) \leqq 2 n-s$ and this implies $\alpha^{t+(n-k)}\left(w_{0}\right) \subset_{e^{1 / 2}} A_{s+3 n}$.)

We infer

$$
\begin{aligned}
\left|\left(\psi_{n} \circ \varphi_{n}\right)\left(u^{m} \alpha^{t}\left(\chi_{\Omega_{j}}\right)\right)-u^{m} \alpha^{t}\left(\chi_{\Omega_{j}}\right)\right|_{2} & \leqq\left(1-\frac{(2 C-2) N}{2 n+1}\right)+\frac{(2 C-2) N}{2 n+1} \varepsilon^{1 / 2} \\
& \leqq \frac{2 N+1}{2 C N+1}+\varepsilon^{1 / 2} \leqq 2 C^{-1}+\varepsilon^{1 / 2}
\end{aligned}
$$

Choosing $C$ sufficiently large and $\varepsilon$ sufficiently small we'll have

$$
2 C^{-1}+\varepsilon^{1 / 2}<\delta
$$

On the other hand

$$
\begin{aligned}
\operatorname{rank}\left(\left(B\left(l^{2}([-n, n])\right) \otimes \mathscr{A}\right)\right) & =(2 n+1) \operatorname{dim} \mathscr{A} \\
& \leqq(2 n+1)(1+\exp ((s+3 n)(h(T)+\varepsilon))) .
\end{aligned}
$$

Clearly

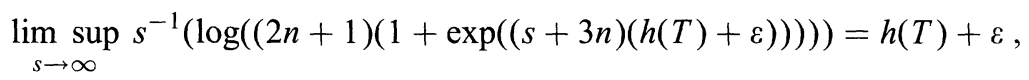

so that

$$
h c p a_{\tau}\left(\operatorname{Ad} u, w_{N} ; \delta\right) \leqq h(T)+\varepsilon .
$$

Since $\delta>0$ and $\varepsilon>0$ are arbitrary we infer

$$
h c p a_{\tau}\left(\operatorname{Ad} u, w_{N}\right) \leqq h(T) .
$$

The conclusion follows.

In view of Proposition 3.6 and of the inequality $h(T) \leqq h(\operatorname{Ad} u)$ we have Corollary. $h(T)=h($ Ad $u)$. 


\section{References}

1. Choi, M.-D., Effros, E.G.: Nuclear $C^{*}$-algebras and the approximation property. Am. J. Math. 100, 61-79 (1978)

2. Christensen, E.: Subalgebras of finite algebras. Math. Ann. 243, 17-29 (1973)

3. Connes, A., Narnhofer, H., Thirring, W.: Dynamical entropy of $C^{*}$-algebras and von Neumann algebras. Commun. Math. Phys. 112, 691-719 (1987)

4. Connes, A., Störmer, E.: Entropy of $\mathrm{II}_{1}$ von Neumann algebras. Acta Math. 134, 289-306 (1975)

5. Hudetz, T.: Topological entropy for appropriately approximated $C^{*}$-algebras. Preprint

6. Kolmogorov, A.N., Tihomirov, B.: $\varepsilon$-entropy and $\varepsilon$-capacity of sets in functional spaces. Uspekhi Mat. Nauk 14, Part 2 (86) 3-86 (1959)

7. Narnhofer, H.: Dynamical entropy for the quantized cat map. Preprint, February 1993

8. Peterson, K.: Ergodic Theory. University Press, 1983

9. Sauvageot, J.-L., Thouvenot, P.: Une nouvelle definition de l'entropie dynamique des systemes non-commutatifs. Commun. Math. Phys. 145, 411-423 (1992)

10. Shannon, C.E., Weaver, W.: The Mathematical Theory of Communications. University of Illinois Press, 1963

11. Störmer, E.: Entropy in operator algebras. Preprint (1992)

12. Störmer, E., Voiculescu, D.: Entropy of Bogoliubov automorphisms of the canonical anticommutation relations. Commun. Math. Phys. 133, 521-542 (1990)

13. Thomsen, K.: Topological entropy for endomorphisms of local $C^{*}$-algebras. Preprint

14. Voiculescu, D.: Alternative entropies in operator algebras. To appear in the Proceedings of the 1992 European Congress of Mathematicians

15. Narnhofer, H., Störmer, E., Thirring, E.: $C^{*}$-dynamical systems for which the tensor product formula for entropy fails. Preprint, Summer 1994

Communicated by A. Connes 
